ARTICLE

Check for updates

https://doi.org/10.1038/s41467-021-26489-0

\title{
Somatic driver mutation prevalence in 1844 prostate cancers identifies ZNRF3 loss as a predictor of metastatic relapse
}

Michael Fraser ${ }^{1,2,3 凶}$, Julie Livingstone (1) ${ }^{4,5,6,7}$, Jeffrey L. Wrana (10) ${ }^{8,9}$, Antonio Finelli ${ }^{1,2,10}$, Housheng Hansen $\mathrm{He}^{10,11}$, Theodorus van der Kwast (10) 12,13, Alexandre R. Zlotta1,2,8,9, Robert G. Bristow (iD ${ }^{11,14,15}$ \& Paul C. Boutros (1) 4,5,6,7,11,16凶

Driver gene mutations that are more prevalent in metastatic, castration-resistant prostate cancer (mCRPC) than localized disease represent candidate prognostic biomarkers. We analyze 1,844 localized (1,289) or MCRPC (555) tumors and quantify the prevalence of 113 somatic driver single nucleotide variants (SNVs), copy number aberrations (CNAs), and structural variants (SVs) in each state. One-third are significantly more prevalent in mCRPC than expected while a quarter are less prevalent. Mutations in $A R$ and its enhancer are more prevalent in $\mathrm{MCRPC}$, as are those in TP53, MYC, ZNRF3 and PRKDC. ZNRF3 loss is associated with decreased ZNRF3 mRNA abundance, WNT, cell cycle \& PRC1/2 activity, and genomic instability. ZNRF3 loss, RNA downregulation and hypermethylation are prognostic of metastasis and overall survival, independent of clinical and pathologic indices. These data demonstrate a strategy for identifying biomarkers of localized cancer aggression, with ZNRF3 loss as a predictor of metastasis in prostate cancer.

\footnotetext{
${ }^{1}$ Department of Surgery, Division of Urology, University of Toronto, Toronto, ON, Canada. ${ }^{2}$ Department of Surgical Oncology, Genitourinary Site Group, Princess Margaret Cancer Centre, Toronto, ON, Canada. ${ }^{3}$ Ontario Institute for Cancer Research, Toronto, ON, Canada. ${ }^{4}$ Department of Human Genetics, University of California, Los Angeles, CA, USA. ${ }^{5}$ Department of Urology, University of California, Los Angeles, CA, USA. ${ }^{6}$ Institute for Precision Health, University of California, Los Angeles, CA, USA. ${ }^{7}$ Jonsson Comprehensive Cancer Centre, University of California, Los Angeles, CA, USA. ${ }^{8}$ Lunenfeld Research Institute, Mount Sinai Hospital, Toronto, ON, Canada. ${ }^{9}$ Mount Sinai Hospital, Sinai Health System, Toronto, Canada. ${ }^{10}$ Princess Margaret Cancer Centre, Toronto, ON, Canada. ${ }^{11}$ Department of Medical Biophysics, University of Toronto, Toronto, ON, Canada. ${ }^{12}$ Department of Laboratory Medicine and Pathobiology, University of Toronto, Toronto, ON, Canada. ${ }^{13}$ Laboratory Medicine Program, University Health Network, Toronto, ON, Canada. ${ }^{14}$ Manchester Cancer Research Centre, Manchester, UK. ${ }^{15}$ University of Manchester, Manchester, UK. ${ }^{16}$ Department of Pharmacology \& Toxicology, University of Toronto, Toronto, ON, Canada. ${ }^{凶}$ email: michael.fraser@cpcgene.com; PBoutros@mednet.ucla.edu
} 
W hile the vast majority of prostate cancers are organconfined at diagnosis ${ }^{1}$, a significant proportion of these tumors relapse following surgery or radiation therapy ${ }^{2,3}$. This necessitates salvage therapy to prevent or limit the development of distant metastases ${ }^{4}$. For example, up to $20 \%$ of men with intermediate-grade prostate cancer will experience biochemical relapse within three years of definitive local therapy ${ }^{5,6}$, which portends an aggressive clinical course. There is therefore a clear need to improve on current risk-stratification guidelines, which rely on three clinical prognostic factors (i.e. Gleason/ISUP grade, pre-treatment serum concentration of prostate-specific antigen (PSA), and clinical T category).

Many somatic mutations have been proposed to predict relapse of localized prostate cancer ${ }^{7-14}$. However, the majority of these studies focus on weak surrogates of disease-specific mortality (e.g. biochemical relapse; BCR). Moreover, while genome-wide analysis offers an unbiased approach to biomarker discovery, discovering, characterizing, and validating these biomarkers is limited by the high false discovery rate that results from simultaneous testing of multiple mutations for association with clinical outcome. Mutations associated with the lethal disease are evolutionarily selected for localized tumors ${ }^{15}$. This suggests that mutations that are highly prevalent in lethal metastatic, castration-resistant prostate cancer (mCRPC) but rare in localized disease may be prognostic biomarkers that reflect the elevated risk of occult metastatic disease. While there are some data comparing the prevalence of driver mutation prevalence in localized disease vs. $\mathrm{mCRPC}^{16,17}$, a comprehensive analysis of the clinical impact of this differential is lacking.

To address these gaps, we quantify the prevalence of 113 mutation types in 72 established prostate cancer driver genes or recurrently mutated genomic regions 7,18 in 1844 patients with either localized prostate cancer or mCRPC. We identify the differential prevalence of seventy-three established driver mutations, including mutations in the androgen receptor and its enhancer region. Amongst these differentially prevalent driver mutations, twenty-four are present in at least $5 \%$ of localized cancers and four are significantly associated with metastatic relapse of localized disease, including genomic gains in $M Y C$ and CCND1. In addition, genomic loss of the WNT pathway inhibitor ZNRF3 is associated with WNT pathway activity and predicts biochemical and metastatic relapse and overall survival in localized prostate cancer. These results demonstrate a method of identifying candidate prognostic genomic biomarkers by comparing primary and metastatic disease and establish ZNRF3 loss as a predictor of aggressive localized prostate cancer.

\section{Results}

Molecular drivers of localized and metastatic prostate cancer. We collected somatic SNV, CNA, and SV calls from eleven DNA sequencing studies of prostate cancer comprising 1844 patients (1289 localized, 555 metastatic; Fig. S1 and S2 and Supplementary Data 1$)^{7,11-15,18-22}$. We curated a list of 113 mutation types-31 CNAs, 43 coding SNVs, 6 non-coding SNVs and 33 SVs-from those identified as either putative drivers or recurrently mutated in two large whole-genome sequencing studies of prostate cancer ${ }^{7,18}$. These encompassed 72 individual genes or genomic loci (Supplementary Data 2).

The most common mutation in localized prostate cancers was loss of NKX3-1, in 644/1279 cases (50.4\%; Fig. 1 and Supplementary Data 3). Other mutations present in at least $20 \%$ of localized cancers were ERG SVs (78/201 cases; $38.8 \%$ ), PTEN SVs (45/201 cases; 22.4\%), MYC gains (267/1279 cases; $20.9 \%$ ), and CDH1 losses (256/1279 cases; $20.0 \%)$. The most common mutation in mCRPC was gain of the androgen receptor
$(A R)$, which occurred in 395/555 cases (71.2\%; Fig. 1 and Supplementary Data 3). Consistent with the higher rate of mutation reported in MCRPC relative to localized disease ${ }^{16}, 25$ genes were mutated in at least $20 \%$ of mCRPC cases, compared with only 4 in localized prostate cancer (Fig. 1 and Supplementary Data 3).

The proportion of localized prostate cancer and $\mathrm{MCRPC}$ cases harboring each driver mutation is shown in Fig. 2A. To assess which mutations are more prevalent in each disease state, we first evaluated the difference in these proportions ('observed $\Delta$ proportion'). Because of the differences in mutational burden between localized disease and $\mathrm{mCRPC}^{18,22}$, we also derived an expected $\Delta$ proportion based on 100,000 samples of the binomial distribution, per driver gene mutation, per sample, weighted by mutational burden and gene size, in each tumor sample (Fig. 2B; see "Methods"). Finally, we computed the difference between observed $\Delta$ proportion and expected $\Delta$ proportion, yielding an adjusted $\Delta$ proportion which indicates whether a driver mutation is prevalent in mCRPC more than expected (adjusted $\Delta$ proportion $>0$ ) or less than expected (adjusted $\Delta$ proportion $<$ 0 ), based on background global mutation burden.

Overall, $37.2 \%$ of driver gene mutations (42/113) were significantly more prevalent than expected in mCRPC $(q<0.05$, adjusted Fisher's Exact test; Fig. $2 \mathrm{C}$ and Supplementary Data 3$)^{21,23}$. By comparison, $27.4 \%$ (31/113) were significantly less prevalent. Driver mutations more prevalent in mCRPC may either provide a selective advantage to metastasis when localized or may result from adaptation to metastatic niches and response to therapy. The largest adjusted $\Delta$ proportion was for genomic gain of the AR locus (gene body or enhancer), which was present in 395/ 555 (71.2\%, 95\% CI: 67.4-74.9) mCRPCs but only 2/1279 (0.16\%; $95 \%$ CI: $0-0.37$ ) localized cancers (adjusted $\Delta$ proportion $=0.609$, 95\% CI: 0.587-0.632, $q=2.35 \times 10^{-5}$, adjusted Fisher's Exact test; Fig. 2C). SNVs in $A R$ were less common overall and were not observed in localized disease (76/555 vs. 0/1204; adjusted $\Delta$ proportion $=0.128,95 \%$ CI: $0.113-0.144, q=2.35 \times 10^{-5}$, adjusted Fisher's Exact test). CNAs in BRCA2 were frequently observed and significantly more prevalent in mCRPC than expected $(219 / 555$ vs. $72 / 1279$; adjusted $\Delta$ proportion $=0.232$, 95\% CI: $0.212-0.251, q=2.35 \times 10^{-5}$, adjusted Fisher's Exact test), consistent with reports showing that germline and somatic aberrations in BRCA2 are more prevalent in $\mathrm{mCRPC}$ than localized disease ${ }^{24,25}$. CNAs and SNVs in TP53 were both significantly more prevalent in mCRPC (SNV: 205/555 vs. 49/ 1204; adjusted $\Delta$ proportion $=0.305,95 \%$ CI: $0.284-0.332$, $q=2.35 \times 10^{-5}$; CNA: $336 / 555$ vs. $156 / 1279$; adjusted $\Delta$ proportion $=0.426,95 \%$ CI: $0.403-0.448, q=2.35 \times 10^{-5}$, adjusted Fisher's Exact test). Similarly, SVs affecting TP53 were more prevalent in metastatic disease (adjusted $\Delta$ proportion $_{\mathrm{SV}}=0.074$, 95\% CI: 0.044-0.104, $q=0.060$, adjusted Fisher's Exact test). While this did not reach our pre-set statistical significance threshold, it is important to note the lower power to detect differences in SV prevalence, relative to other mutational classes. Consistent with previous reports $16,26, S P O P$ SNVs were less prevalent in MCRPC $^{16}$ (32/555 vs. 103/1204; adjusted $\Delta$ proportion $=-0.051,95 \%$ CI: $-0.061-0.041 ; q=2.35 \times 10^{-5}$, adjusted Fisher's Exact test).

Candidate genomic biomarkers of aggressive localized prostate cancer. Relapse of localized prostate cancer following curativeintent therapy is due, at least in part, to the presence of occult metastatic disease at initial presentation. We hypothesized that mutations prevalent in mCRPC than localized disease might be prognostic for relapse of localized prostate cancer. From the list of 73 mutations with significantly different prevalence in mCRPC 

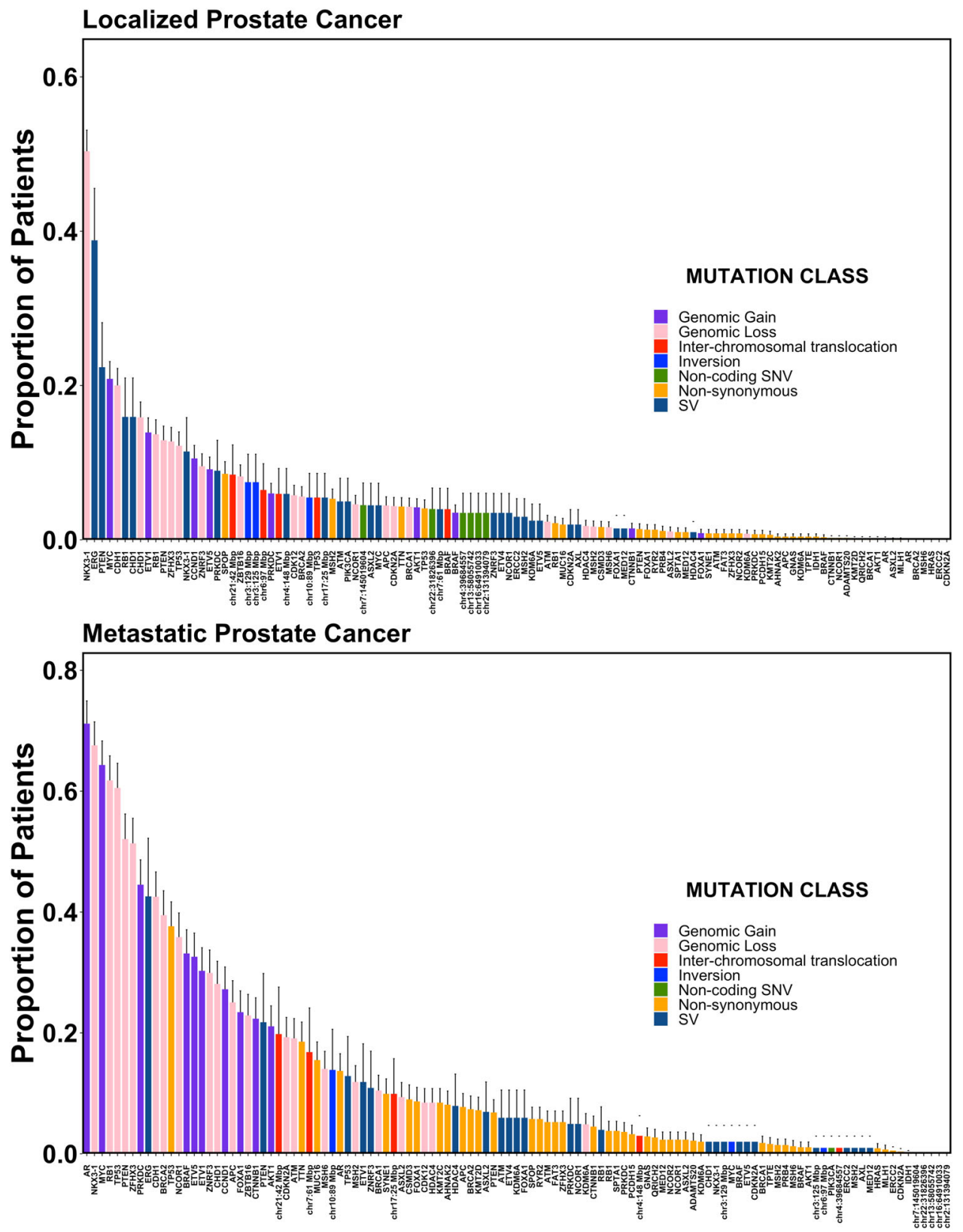

Fig. 1 Frequency distribution of genes affected by driver mutations in prostate cancer. The full cohort $(n=1844)$ was split into localized $(n=1289)$ and metastatic $(n=555)$ disease. Estimated proportion of patients in each cohort harboring each of 113 specific driver mutations. $X$-axis indicates the gene or genomic locus affected. Bar color indicates the type of driver mutation affecting that gene. Several genes are affected by multiple mutation types (e.g. TP53, PTEN, and others). Bars show the proportion of patients harboring the indicated mutation. Error bars represent $95 \%$ confidence intervals. Centre of the error bars represent the observed proportion. Source data are provided as a Source Data file.

vs. localized disease ( 42 with increased prevalence, 31 with lower prevalence), we identified 24 that occurred in at least $5 \%$ of all localized cancers evaluated (Supplementary Data 4). These 24 driver mutations comprised 16 CNAs, 6 SVs, and 2 SNVs. We then used univariable Cox proportional hazards modeling to assess whether any of these 24 mutations are associated with metastatic relapse of localized disease. Using patient outcome data from the CPCG study ( $n=376), 4$ of the 24 driver mutations were both more prevalent in $\mathrm{MCRPC}$ and associated with significantly higher risk of metastatic relapse $(q<0.05$; Table $S 1)$ : $M Y C, C C N D 1$, and PRKDC gain \& ZNRF3 loss. Three other mutations-losses of CDK12, ETV5, and TP53-were associated with metastatic relapse but did not reach our pre-determined level of statistical significance $(q<0.05)$. PTEN and RB1 loss were not prognostic of metastatic relapse (although PTEN loss was associated with biochemical relapse in the CPCG cohort; $p=0.035$, log-rank test). In a multivariable Cox proportional hazards model including all seven driver CNAs with $q \leq 0.25$, ZNRF3 loss, MYC gain, CCND1 gain, and CDK12 loss remained significantly associated with risk of metastatic relapse (Fig. S3).

To confirm these associations with adverse outcome, we employed two independent validation cohorts: TCGA PRAD ${ }^{19}$ and MSKCC ${ }^{27}$ localized prostate cancer cohorts. In TCGA, MYC and CCND1 gains as well as ZNRF3 losses were prognostic for progression-free survival (Table S2); on multivariable analysis, only ZNRF3 remained prognostic (Table S2). Similarly, these CNAs were also prognostic for poor outcomes in the MSKCC cohort (Tables S3, S4). Taken together, these data support the 


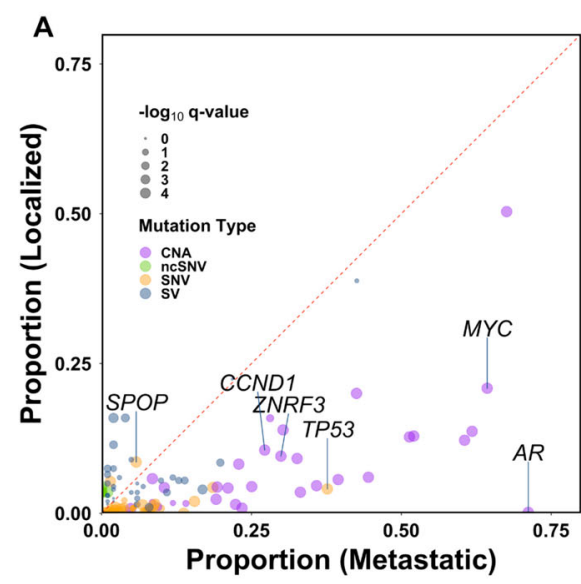

C

B
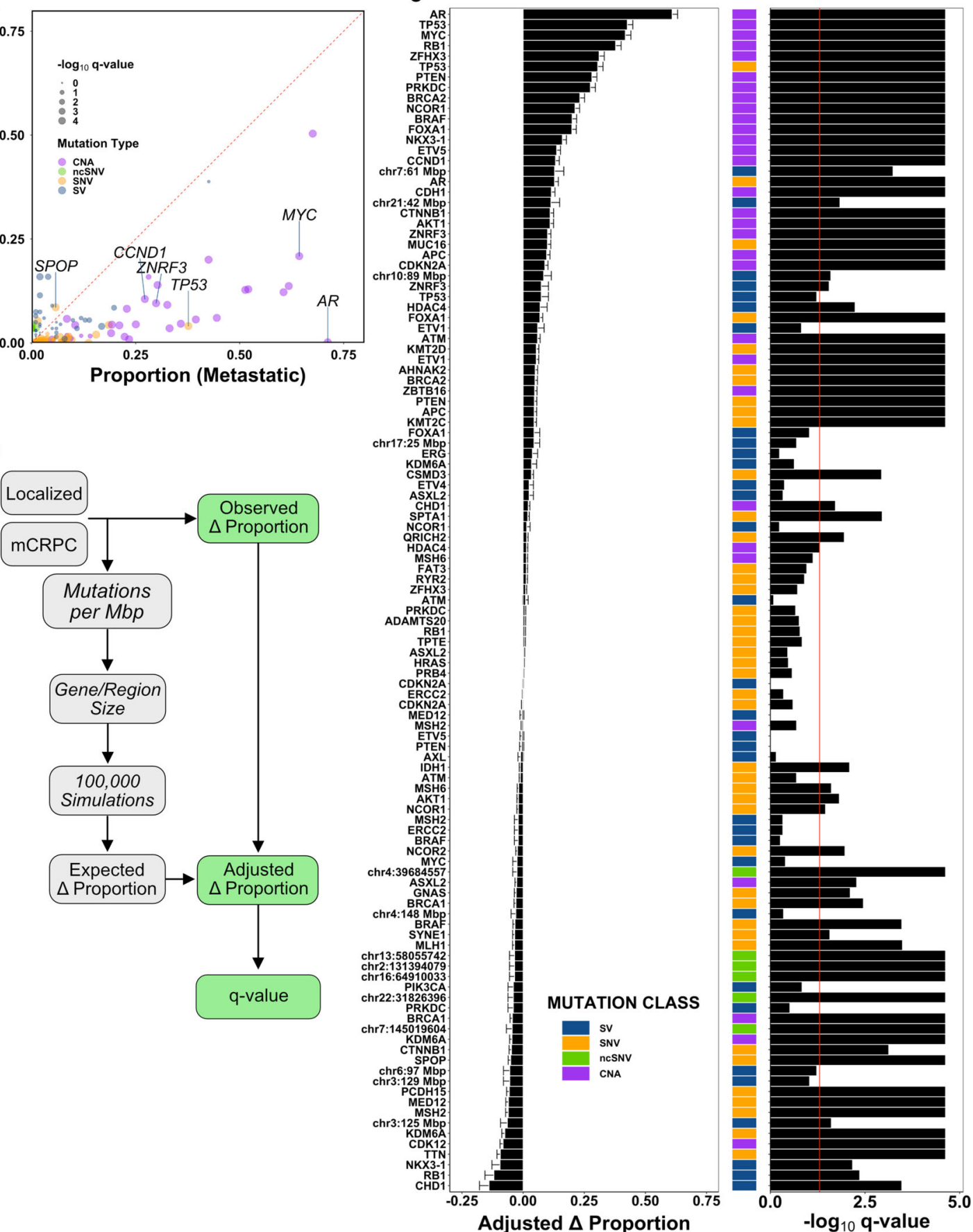

Fig. 2 Prevalence of driver gene mutations in localized and metastatic prostate cancer. A The proportion of tumors harboring each driver mutation in localized prostate cancer or mCRPC ('Observed $\Delta$ Proportion'), as described. Dot size indicates $-\log _{10} q$-value; dot color indicates driver mutation type. Specific genes of interest are labeled. B Comparison of driver gene mutation prevalence in localized disease and mCRPC. Differences in proportion of localized and metastatic cases harboring each driver mutation ('Observed $\Delta$ Proportion') were subtracted from the difference in proportions resulting from 100,000 simulations, per gene, per sample, where the probability of observing a mutation in a given sample was weighted by the global mutational burden (i.e. SNVs per Mb or PGA) in that sample ('Expected $\Delta$ Proportion') to generate the Adjusted $\Delta$ Proportion. A two-sided $p$-value was calculated as the proportion of simulated proportions that were as extreme or more extreme than the observed $\Delta$ proportion. $q$-values were then derived using the False Discovery Rate method. C Positive adjusted $\Delta$ proportion indicates higher than expected prevalence in $\mathrm{mCRPC}$, while negative adjusted $\Delta$ proportion indicates lower than expected prevalence. Mutations are ordered from top to bottom by adjusted $\Delta$ proportion, as shown by the height of each bar. Error bars represent $95 \%$ confidence intervals; centre of the error bars represents the adjusted $\Delta$ proportion. Statistical significance was tested using adjusted Fisher's Exact tests with correction for multiple testing using the False Discovery Rate method. Mutations with $q$-value $<0.05$ (red line) were considered statistically significant. Source data are provided as a Source Data file. 
utility of this approach to prognostic biomarker discovery. MYC, CCND1, and PRKDC have previously been linked to adverse clinical outcomes in prostate cancer ${ }^{28-31}$, and thus serve as positive controls. ZNRF3 loss has been reported in five mCRPC patients ${ }^{18,22}$ (two monoallelic and three biallelic) and several studies have observed losses in the region containing ZNRF3 $(\operatorname{chr} 22 \mathrm{q} 12)^{7,19,27,32,33}$. To the best of our knowledge, however, ZNRF3 loss has not previously been explicitly reported as a recurrent CNA in localized prostate cancer or associated with adverse clinical outcomes in localized disease.

ZNRF3 genomic loss is associated with gene expression and clinical features. We next focused on the functional and clinical implication of ZNRF3 loss. ZNRF3 loss was identified in 166/555 metastases (29.9\%; 95\% CI: 26.1-33.7) and 122/1279 localized cancers $(9.54 \%$; $95 \%$ CI: $7.93-11.1$; adjusted $\Delta$ proportion $=0.10$, 95\% CI: $0.086-0.113, q=2.35 \times 10^{-5}$, adjusted Fisher's Exact test). While ZNRF3 loss was present in $\sim 10 \%$ of localized cancers overall, this CNA was enriched in aggressive disease. In CPCGENE, $25 \%$ of patients $(9 / 36)$ who relapsed metastatically harbored ZNRF3 loss while only $5.2 \%$ of patients (18/341) who did not harbor it $\left(\mathrm{OR}=5.98,95 \% \mathrm{CI}: 2.45-14.6 ; p=3.29 \times 10^{-4}\right.$, Fisher's Exact test; Fig. S4A). Similarly, in the TCGA cohort, ZNRF3 loss was identified in $11.9 \%(58 / 489)$ of patients; of the 91/489 patients with disease progression, 20/91 (22.0\%) harbored ZNRF3 loss while only $9.55 \%$ of patients who did not progress harbored this CNA $(\mathrm{OR}=2.67,95 \% \quad \mathrm{CI}: 1.47-4.85$; $p=1.64 \times 10^{-3}$, Fisher's Exact test; Fig. S4B). ZNRF3 loss was also associated with higher grade tumors in both CPCG and TCGA (Fig. S4C). Thus, ZNRF3 loss appears to identify a subtype of localized prostate cancer associated with aggressive clinical outcomes.

ZNRF3 loss was prognostic for BCR and metastatic relapse on univariable Cox proportional hazards analysis in CPCG $\left(\mathrm{HR}_{\mathrm{BCR}}=2.18,95 \% \mathrm{CI}: 1.31-3.64, p=2.87 \times 10^{-3}\right.$, Wald test; $\mathrm{HR}_{\mathrm{METS}}=4.57,95 \%$ CI: $2.12-9.84, p=1.03 \times 10^{-4}$; Wald test; Fig. 3A, B). These effects remained significant after controlling for standard clinical prognostic variables (i.e. pre-treatment PSA, diagnostic ISUP grade group, and clinical $\mathrm{T}$ category) in multivariable Cox proportional hazards modeling $\left(\mathrm{HR}_{\mathrm{BCR}}=1.77\right.$, $95 \%$ CI: $1.05-3.00, p=0.034$, Wald test; $N_{\mathrm{BCR}}=126 / 376$; $\mathrm{HR}_{\text {METS }}=3.01,95 \%$ CI: $1.35-6.74, p=7.32 \times 10^{-3}$, Wald test; $N_{\text {METS }}=36 / 376$; Fig. 3 C, D; univariable Cox models for all clinical prognostic factors are shown in Table S5).

To validate the transcriptomic impact of ZNRF3 genomic loss, we assessed ZNRF3 RNA abundance in 208 CPCG tumors with matched RNA abundance and CNA data. ZNRF3 RNA abundance spans a limited range across tumor specimens (mean $\log _{2}$ RNA abundance $=6.63$, range: 6.09-7.47; Fig. S5). Tumors harboring a genomic loss in ZNRF3 had significantly lower ZNRF3 RNA abundance than ZNRF3 neutral tumors (mean $\log _{2}$ RNA abundance difference $=0.163,95 \%$ CI: $0.132-0.195$, $p=0.021$, Mann-Whitney $\mathrm{U}$ test; Fig. S5). This association validated in TCGA (mean $\log _{2}$ RNA abundance difference $=$ 0.450, 95\% CI: $0.303-0.596, p=4.06 \times 10^{-6}$, Mann-Whitney U test; Fig. S5) and Taylor/MSKCC cohorts (mean $\log _{2}$ RNA abundance difference $=0.075,95 \%$ CI: $0.070-0.079$, $p=1.13 \times 10^{-2}$, Mann-Whitney U test; Fig. S5). Only four patients in the Gerhauser cohort with ZNRF3 loss had available RNA abundance data, but there was a trend toward decreased ZNRF3 RNA abundance relative to ZNRF3 neutral patients (mean $\log _{2}$ RNA abundance difference $=1.37,95 \%$ CI: -1.42 to 4.16, $p=0.11$, Mann-Whitney U test; Fig. S5).

ZNRF3 RNA abundance was inversely associated with ISUP grade in four of five independent cohorts of localized prostate cancer cohorts (Fig. S6). Likewise, in CPCG, ZNRF3 RNA abundance was inversely associated with risk of BCR on multivariable analysis controlling for clinical ISUP grade, pretreatment PSA, and clinical T-category $\left(N_{\mathrm{BCR}}=46 / 205 ; \mathrm{HR}_{\mathrm{BCR}}=\right.$ 0.22 , 95\% CI: $0.06-0.86, p=0.030$, Wald test; Fig. S7A). ZNRF3 RNA abundance was not significantly associated with metastatic relapse on multivariable analysis, potentially due to a lack of statistical power resulting from the low event rate in this intermediate risk cohort $\left(N_{\text {METS }}=16 / 205\right.$ patients; $\mathrm{HR}_{\text {METS }}=$ $0.20, p=0.18$, Wald test). ZNRF3 RNA abundance remained inversely associated with BCR risk after controlling for ZNRF3 loss in multivariable Cox proportional hazards analysis $\left(\mathrm{HR}_{\mathrm{BCR}}=\right.$ $0.21,95 \%$ CI: $0.05-0.78, p=0.02$, Wald test; $N_{\mathrm{BCR}}=46 / 205$; Fig. S7B).

We validated this association between ZNRF3 RNA and risk of adverse clinical outcome in three independent cohorts: TCGA, EOPC, and a high risk/high-volume intermediate risk cohort $\left(\mathrm{LTRI}^{34}\right)$, in which low ZNRF3 RNA abundance was associated with a significantly higher risk of PFS, BCR, and metastatic relapse (Fig. S8). In the LTRI cohort, 18/18 patients with low ZNRF3 RNA abundance experienced metastatic relapse within 7 years following surgery, compared with only $2 / 29$ patients with high ZNRF3 RNA abundance $\left(p=4.16 \times 10^{-11}\right.$, Fisher's Exact test; Fig. S8D). ZNRF3 RNA was not related to the risk of disease progression in the Taylor/MSKCC cohort. Taken together, these data demonstrate that genomic loss or low RNA abundance of ZNRF3 preferentially occur in aggressive localized prostate cancer, independent of standard clinical prognostic factors.

ZNRF3 loss predicts poor outcome localized prostate cancer. We recently developed a six-feature clinico-genomic signature that predicts biochemical relapse in men with localized prostate cancer7. To assess whether ZNRF3 genomic loss adds independent prognostic value to these features, we stratified the CPCG cohort based on both signature features and ZNRF3 loss. Overall, 298/379 (78.6\%) CPCG cases had informative data for the six features in the signature (MYC gain, ATM SNVs, TCERGL1 hypomethylation, ACTL6B hypermethylation, chr7:61 Mbp interchromosomal translocations, and clinical $\mathrm{T}$ category). On multivariable analysis, both the Fraser signature and ZNRF3 remained prognostic of $\mathrm{BCR}$ and metastasis, after controlling for ISUP grade group, PSA, and clinical $\mathrm{T}$ category $\left(\mathrm{HR}_{\mathrm{BCR}}=1.77\right.$, $95 \%$ CI: $1.06-2.98, p=0.030$, Wald test; $N_{\mathrm{BCR}}=126 / 375$; $\mathrm{HR}_{\mathrm{METS}}=2.86,95 \%$ CI: $1.29-6.34, \quad p=0.019$, Wald test; $N_{\text {METS }}=36 / 375$; Table S6).

In the CPCG cohort, ZNRF3 genomic loss was associated with a significantly higher percentage of the genome altered by a CNA (Mean Adjusted PGA: 6.96\%, 95\% CI: 6.27-7.66 vs. $11.1 \%$, 95\% CI: 7.66-14.5, $p=4.57 \times 10^{-3}$, Mann-Whitney U test; Fig. S9A), and this validated in the TCGA (Mean Adjusted PGA: 13.2\%, $95 \%$ CI: $11.5-15.0$ vs. $30.0 \%, 95 \%$ CI: $24.0-36.1, p=2.11 \times 10^{-10}$, Mann-Whitney U test; Fig. S9B) and Taylor/MSKCC cohorts (Mean Adjusted PGA: 27.8\%, 95\% CI: $14.2-41.4$ vs. 9.45\%, 95\% CI: 7.51-11.4, $p=6.40 \times 10^{-4}$, Mann-Whitney U test; Fig. S9C). Despite this, ZNRF3 genomic loss remained prognostic of metastatic relapse in the CPCG cohort after correcting for adjusted PGA, PSA, ISUP grade, clinical $\mathrm{T}$ category, and intraductal carcinoma of the prostate/cribriform architecture (IDC-P/CA) - an established negative prognostic factor ${ }^{35,36}$ in multivariable Cox proportional hazards models $\left(\mathrm{HR}_{\mathrm{METS}}=3.53\right.$, $95 \%$ CI: $1.47-8.47, p=4.72 \times 10^{-3}$, Wald test; $N_{\text {METS }}=30 / 326$; Table 1). In contrast, ZNRF3 loss was not prognostic of BCR after correcting for these clinical and pathologic prognostic features $\left(\mathrm{HR}_{\mathrm{BCR}}=1.49,95 \% \mathrm{CI}: 0.83-2.67, p=0.180\right.$, Wald test; $N_{\mathrm{BCR}}=$ $106 / 326)$. These results support the hypothesis that ZNRF3 loss is 
A

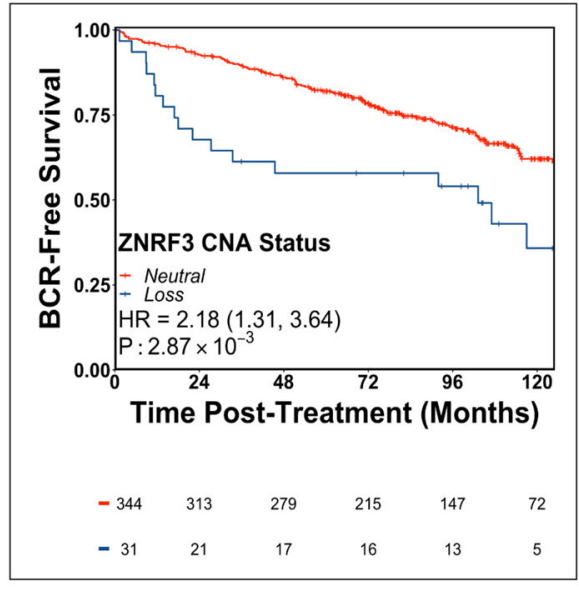

C

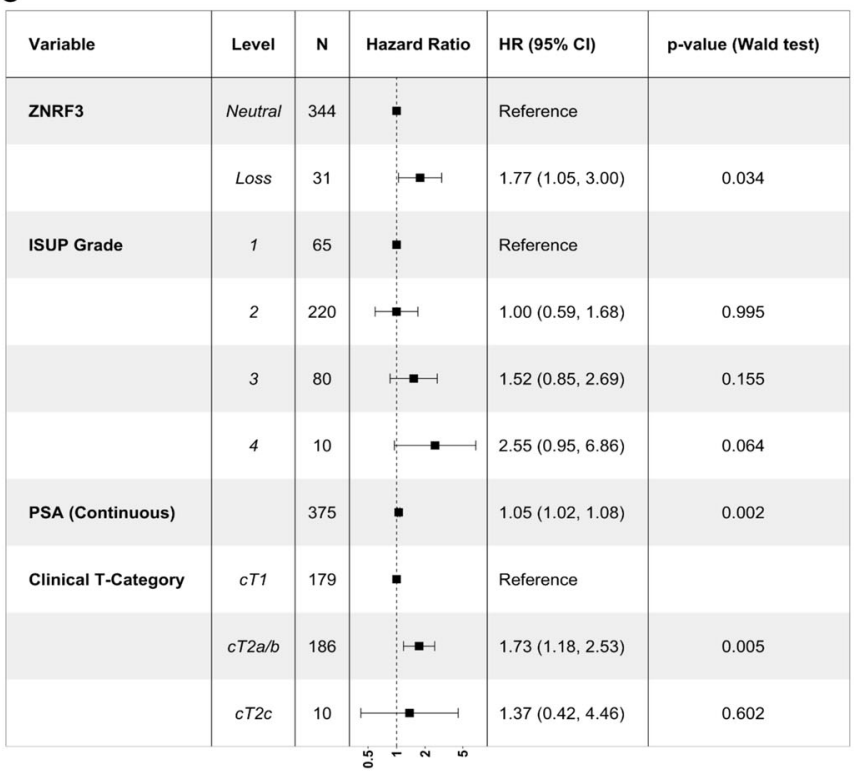

D

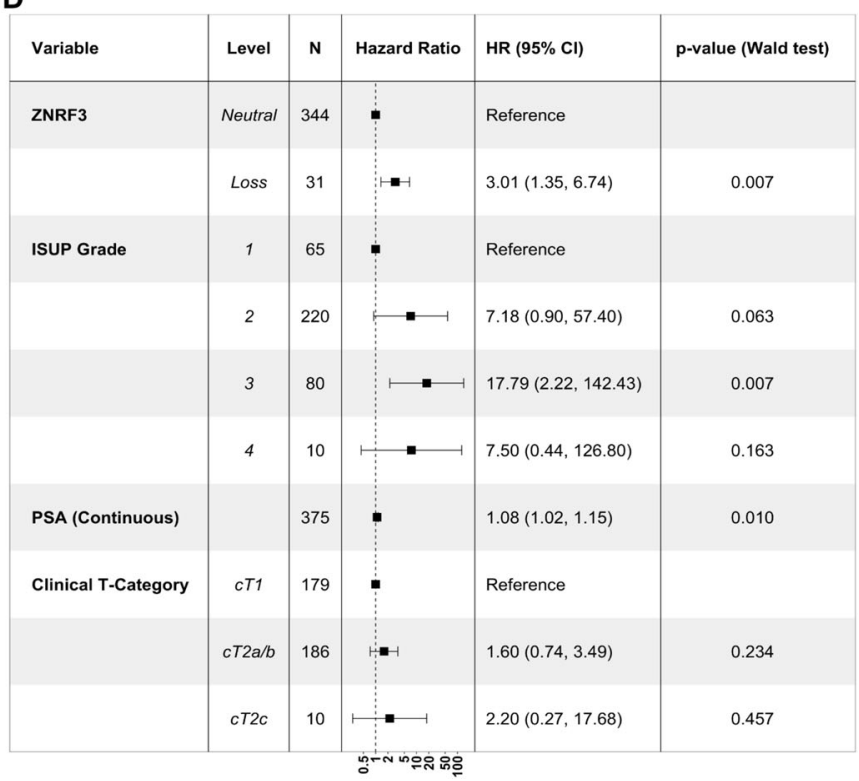

Fig. 3 ZNRF3 genomic loss is an independent prognostic factor for aggressive localized prostate cancer. Biochemical relapse-free rate (A) and metastatic relapse-free rate (B; mRFR) in CPCG patients with tumor specimens with (blue) or without (red) genomic loss of ZNRF3. Two-sided p-values generated from a Wald test. C, D Forest plots of multivariable Cox proportional hazards analyses of ZNRF3 CNA status with clinical prognostic factors for biochemical relapse (C) and metastatic relapse (D). Error bars indicate $95 \%$ confidence interval of the reported hazard ratio. Source data are provided as a Source Data file.

an independent predictor of metastatic relapse in localized prostate cancer.

ZNRF3 loss occurred in the context of a relatively large region of genomic loss on chromosome 22q12.1 (median deletion: 6.0 Mbp, range: $1.01-35.1 \mathrm{Mbp}$ ) and the smallest number of genes co-deleted with ZNRF3 was 10 (median co-deleted genes =90), covering $1.31 \mathrm{Mbp}$. All 417 genes on chromosome 22 were codeleted with ZNRF3 in at least one patient. Of these, 29 (5.3\%) were significantly associated with metastatic relapse in CPCG $(q<0.01$, Wald test; Table S7). Of these 29 genes co-deleted with ZNRF3 and associated with metastasis, 9/29 also showed a significant reduction in RNA abundance in cases with genomic loss in this region $(q<0.05$, Mann-Whitney $\mathrm{U}$ test), and thus represent likely candidate drivers of the aggressive phenotype associated with this deletion. To further refine this gene list and help to identify the driver of aggression in this region, we next determined the association between RNA abundance of these 9 genes and adverse clinical outcomes in four independent cohorts (CPCG, TCGA, EOPC, and LTRI) using univariable Cox proportional hazards models (Table S8). The only gene with RNA abundance significantly associated with adverse outcome across all four cohorts $(q<0.05)$ was $Z N R F 3$. These data are thus consistent with the hypothesis that ZNRF3 loss drives clinical aggression of localized prostate cancer.

Molecular Hallmarks of ZNRF3 genomic loss. To further understand the functional correlates of ZNRF3 in prostate 


\begin{tabular}{|c|c|c|c|c|c|}
\hline Variable & Level & $\mathbf{N}$ & Hazard Ratio & 95\% Confidence Interval & $p$-Value \\
\hline \multicolumn{6}{|l|}{ ZNRF3 Loss } \\
\hline & Neutral & 298 & - & - & - \\
\hline & Loss & 28 & 3.53 & $1.47-8.47$ & $4.72 \mathrm{E}-03$ \\
\hline \multirow{4}{*}{$\begin{array}{l}\text { PSA } \\
\text { Gleason Score }\end{array}$} & Continuous & 326 & 1.05 & $0.96-1.14$ & 0.31 \\
\hline & $3+4$ & 190 & 4.29 & $0.53-34.6$ & 0.17 \\
\hline & $4+3$ & 73 & 9.62 & $1.15-80.3$ & 0.036 \\
\hline & $4+4$ and above & 9 & 5.33 & $0.31-93.0$ & 0.25 \\
\hline \multicolumn{6}{|c|}{ Clinical T Category } \\
\hline & cT1 & 157 & - & - & - \\
\hline & $\mathrm{cT} 2 \mathrm{a} / \mathrm{b}$ & 160 & 1.63 & $0.68-3.94$ & 0.27 \\
\hline $\begin{array}{l}\text { Adjusted PGA } \\
\text { IDC-P/CA }\end{array}$ & Present & 104 & 1.58 & $0.67-3.57$ & 0.27 \\
\hline
\end{tabular}

cancers, we determined global RNA abundance patterns related to ZNRF3. To maximize the likelihood of identifying functionally important correlations, we looked for associations with ZNRF3 loss in both the CPCG and TCGA cohorts. Using the Molecular Signatures Database (MSigDB; http://software.broadinstitute.org/ gsea/msigdb/annotate.jsp), we identified 140 genes with a Spearman's $\rho \geq 0.4$ in both cohorts (Supplementary Data 5). Of these, five were located on chromosome 22 , and were therefore excluded from the downstream analyses. The remaining 135 genes were enriched for genes downregulated in metastatic prostate cancer $^{37}\left(13 / 135 ; q=2.2 \times 10^{-7}\right.$, Hypergeometric test) and for genes involved in positive regulation of canonical WNT signaling (GO: 0060070; 9/140, $q=7.57 \times 10^{-4}$, Hypergeometric test); genes implicated in WNT signaling with significantly different RNA abundance in both CPCG and TCGA cases harboring ZNRF3 loss are shown in Table S9. These data suggest that ZNRF3 downregulation in localized prostate cancer may activate WNT signaling, an established driver of mCRPC ${ }^{16,21}$.

Multiple ZNRF3 features are associated with adverse outcomes in localized prostate cancer. The vast majority of ZNRF3 losses are monoallelic. In TCGA, for example, 7/65 (10.8\%) ZNRF3 losses are biallelic, while across both mCRPC cohorts, 7/157 (4.5\%) ZNRF3 losses are biallelic. As such, other mechanisms-including epigenetic silencing-may contribute to the downregulation of ZNRF3 RNA observed in aggressive localized disease. We therefore analyzed global DNA methylation patterns in localized prostate cancers. In both the $\mathrm{CPCG}^{38}$ and TCGA cohorts, the CpG most significantly differentially methylated between patients with and without elevated ZNRF3 RNA was located in the ZNRF3 $5^{\prime}$ promoter region (probe ID: cg11986861; Fig. $4 \mathrm{~A}$ and S11A, B). Methylation of this $\mathrm{CpG}$ was increased by 1.4fold and 1.7-fold in CPCG and TCGA cases with low ZNRF3 RNA abundance, respectively, and was inversely correlated with ZNRF3 RNA abundance (CPCG: $\rho=-0.41, p=3.19 \times 10^{-9}$; Fig. 4B; TCGA: $\rho=-0.50, p=1.12 \times 10^{-31}$; Fig. S11C). Despite the loss of one $Z N R F 3$ allele, this CpG was also significantly hypermethylated in tumors with ZNRF3 loss (CPCG: $p=9.16 \times 10^{-3}$; TCGA: $p=2.48 \times 10^{-3}$, Mann-Whitney U test; Fig. 4C, D). ZNRF3 hypermethylation was associated with increased risk of metastatic relapse in CPCG (HR: 2.18, 95\% CI: 1.06-4.05; $p=3.47 \times 10^{-2}$; Wald test) and of shorter progression-free survival in TCGA (HR: 2.19, 95\% CI:
1.45-3.31; $p=1.83 \times 10^{-4}$; Wald test). ZNRF3 RNA abundance was significantly lower in tumors harboring one or more ZNRF3 alteration (i.e. monoallelic loss, low RNA abundance, and/or hypermethylation; $p=5.42 \times 10^{-34}$, one-way ANOVA; Fig. S11D) and those tumors harboring $>1$ alteration had significantly lower ZNRF3 RNA abundance than those harboring only a single alteration $\left(p=1.78 \times 10^{-3}\right.$, Tukey post-hoc test). In both the CPCG and TCGA cohorts, patients whose tumor genomes harbored more than one ZNRF3-associated feature were at significantly higher risk of adverse outcomes than those whose genomes harbored one or fewer (Fig. 4E, F).

ZNRF3 loss is associated with activation of cell cycle progression and PRC1/2 pathways. We next assessed associations between ZNRF3 loss and clinical, pathological, and genomic features in the CPCG cohort (Fig. 5A). ZNRF3 genomic loss was not associated with age at diagnosis $(q=0.491$, Mann-Whitney $U$ test $)$. Moreover, ZNRF3 genomic loss was not associated with the presence of IDC/ CA, either across the full cohort $(q=0.491$, Fisher's Exact test) or when patients were stratified by ISUP grade group (to account for the increased prevalence of IDC/CA in higher grade tumors ${ }^{39,40}$; ISUP grade group 1: $q>0.99$; ISUP grade group 2: $q=0.43$; ISUP grade groups 3 and 4: $q>0.99$; Fisher's Exact tests). IDC/CA was prognostic of metastatic relapse on univariable analysis $\left(\mathrm{HR}_{\mathrm{METS}}=2.14\right.$, 95\% CI: $1.12-4.52, p=0.046$, Wald test), as previously reported ${ }^{41,42}$. However, on multivariable analysis including ZNRF3 loss, ISUP grade, PSA, clinical T-category, and adjusted PGA $\left(N_{\text {METS }}=30 / 326\right.$; Fig. S10), IDC/CA was not prognostic. ZNRF3 loss was not significantly associated with ETS fusion status (Supplementary Data 6), chromothripsis $(p=0.208$, Fisher's Exact test), kataegis $(q=0.298$, Fisher's Exact test), global SNV burden $(p=0.376$, Mann-Whitney $\mathrm{U}$ test), or tumor hypoxia, measured either using a consensus RNA abundance surrogate signature ${ }^{43}$ ( $p=0.518$, Mann-Whitney U Test) or by direct intratumoral oxygen measurements ${ }^{32,44}(p=0.550$, Mann-Whitney U Test). We observed a significant enrichment of CNAs in APC (loss) or CTNNB1 (gain) in tumors harboring ZNRF3 loss (APC Loss + ZNRF3 Loss: $\mathrm{OR}=2.08, q=1.06 \times 10^{-4}$, Fisher's Exact test; CTNNB1 Gain + ZNRF3 Loss: $\mathrm{OR}=1.92$, $q=2.50 \times 10^{-3}$, Fisher's Exact test; Either CNA + ZNRF3 Loss: $\mathrm{OR}=2.24, q=9.81 \times 10^{-7}$, Fisher's Exact test). SNVs in either APC or CTNNB1 were not associated with ZNRF3 loss (APC: $p=0.843$; 
A

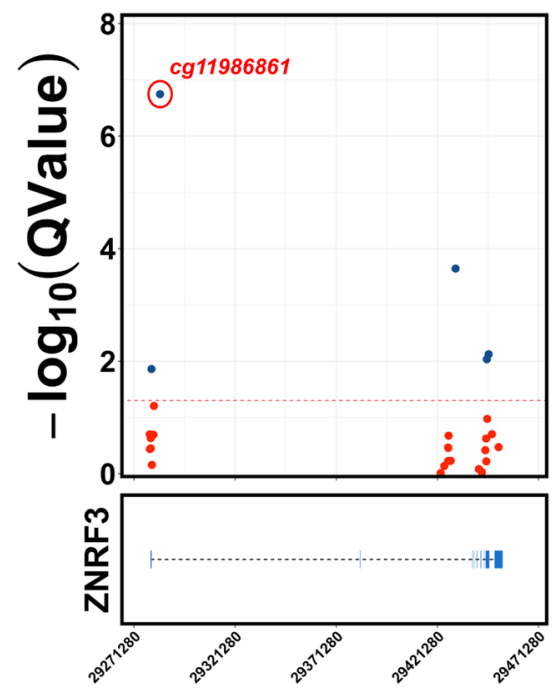

Position (Chromosome 22)

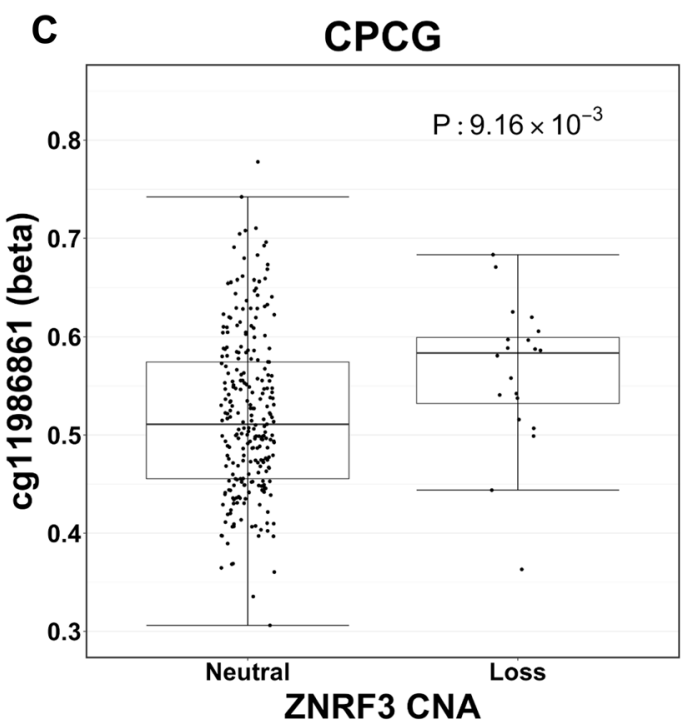

E

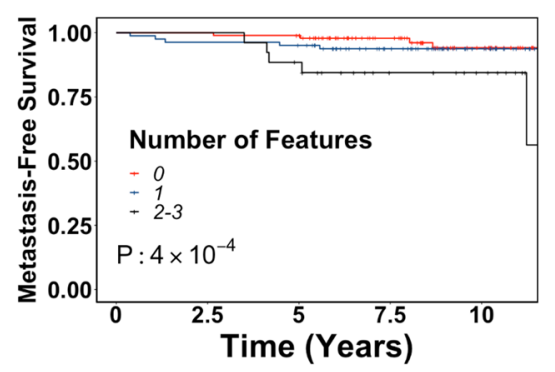

CTNNB1: $q=1$, Fisher's Exact tests). On multivariable analysis, ZNRF3 and APC loss were independently prognostic of PFS in TCGA $\left(N_{\mathrm{PFS}}=91\right)$ and only ZNRF3 loss was independently prognostic of metastatic relapse in CPCG $\left(N_{\mathrm{METS}}=36\right.$; Table S10).

To assess the potential functional role of ZNRF3 loss in localized prostate cancer, we performed Gene Set Enrichment Analysis (GSEA) in tumors with or without ZNRF3 loss. We
B
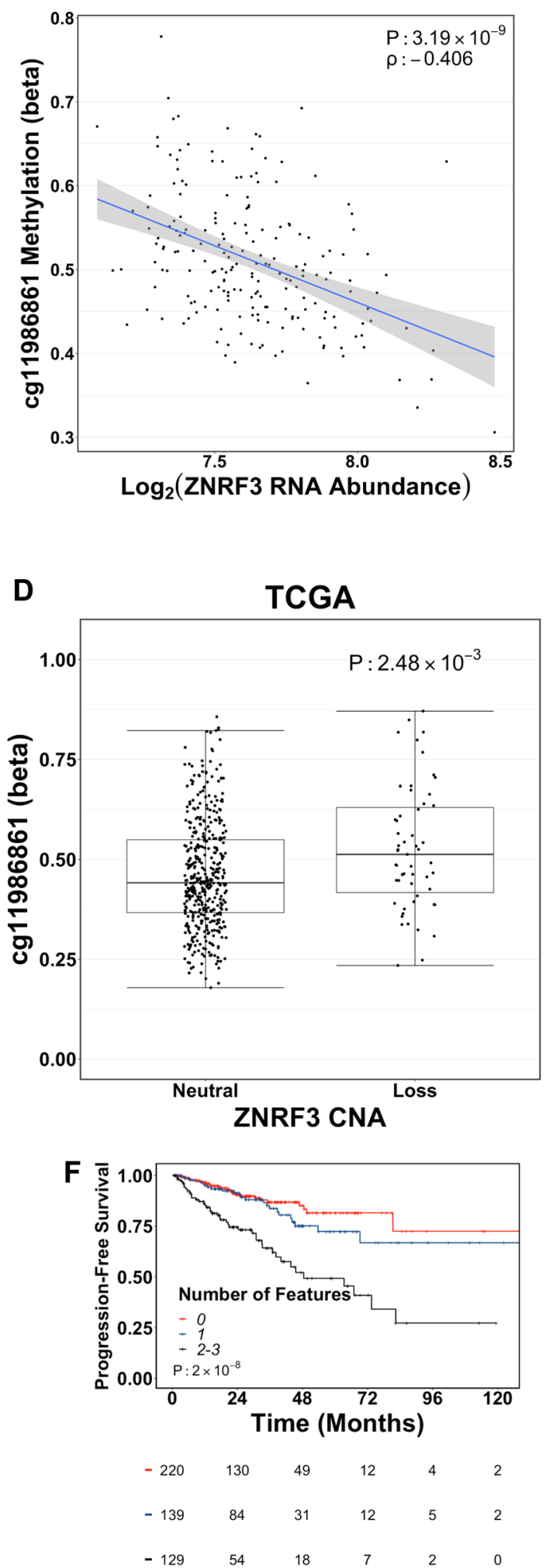

initially focused on TCGA cases to capitalize on the larger number of samples with RNA abundance data $(n=493)$. GSEA of Gene Ontology (GO) pathways revealed enrichment of cell cycle progression gene sets in tumors harboring ZNRF3 loss (Fig. 5B), which validated in two independent cohorts of both localized and metastatic prostate cancer (Fig. S12A, B). Given the enrichment of cell cycle progression gene sets in tumors 
Fig. 4 Aberrant methylation of ZNRF3 in aggressive localized prostate cancer. A The ZNRF3 5' promoter (probe cg11986861) is significantly hypermethylated in localized prostate cancers harboring low ZNRF3 RNA abundance. Coordinates refer to the GRCh37 (hg19) human genome build. Blue dots represent probes with statistically significant differential methylation $(Q<0.05)$. B ZNRF3 RNA abundance is inversely associated with methylation of the ZNRF3 5' promoter. Shaded area shows 95\% confidence interval of the best fit line. Two-sided $p$-value generated from a permutation test. C, D ZNRF3 $5^{\prime}$ promoter methylation is significantly elevated in tumors harboring ZNRF3 allelic losses. $p$-values from a Mann-Whitney U test. CPCG: $n=286$ patients; TCGA: $n=493$ patients. Centre of box represents the median value. Lower and upper box hinges correspond to the first and third quartile. Whiskers extend to the largest and smallest values no further than 1.5-times the Interquartile Range. E, F Patients whose tumors harbor $>1$ aberrant ZNRF3associated feature (i.e. monoallelic loss, low RNA abundance, $5^{\prime}$ promoter hypermethylation) are at significantly higher risk of metastatic relapse (E; CPCG) and disease progression (F; TCGA) than those patients with one or no features. $p$-values from a log-rank test. Colors indicate the number of features present in the tumor. Source data are provided as a Source Data file.

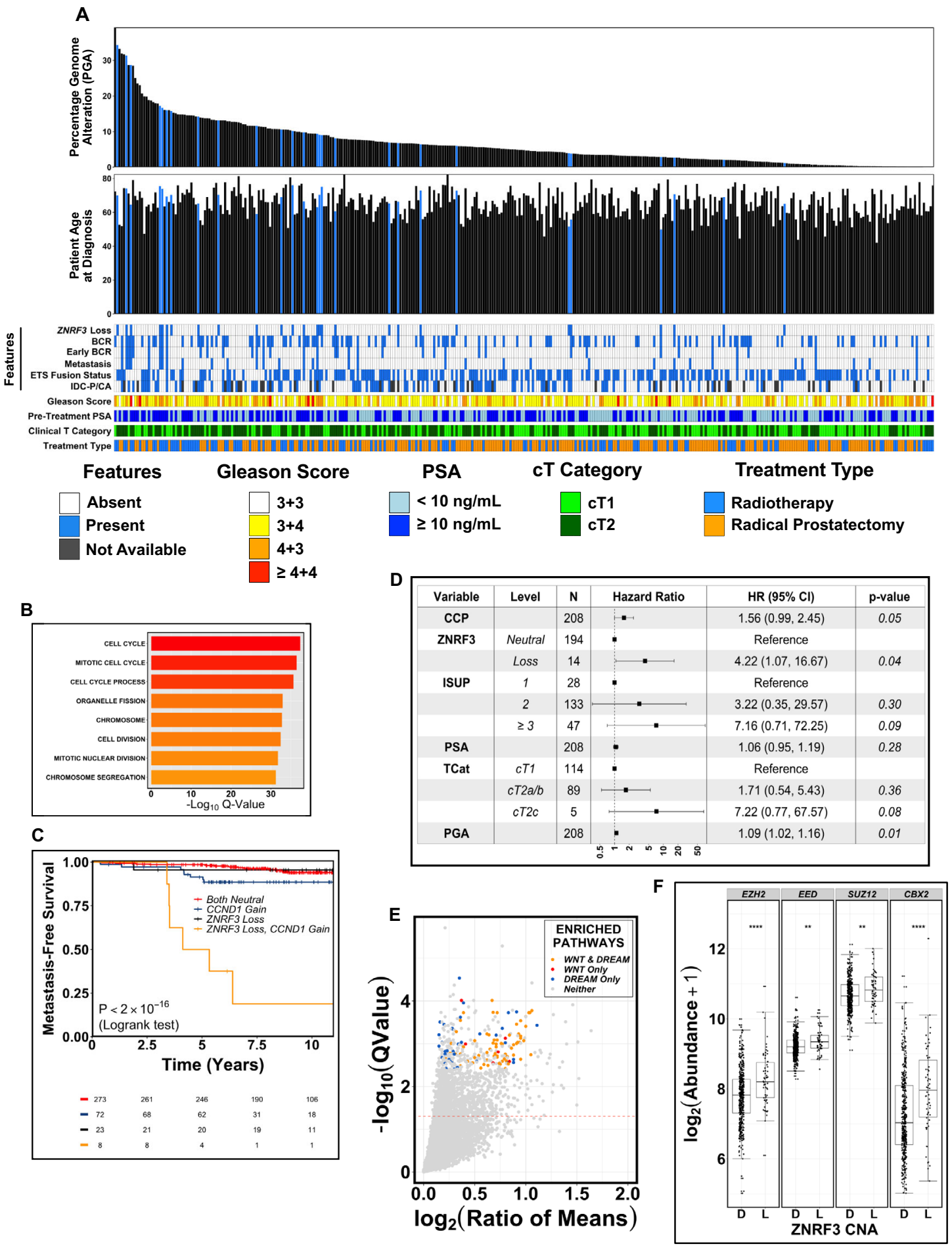


Fig. 5 Molecular and clinical correlates of ZNRF3 genomic loss. A Patients in the CPCG cohort ordered from left to right by percentage of the genome altered by a copy number aberration (PGA). Patient age at diagnosis (years) is also shown. Blue bars correspond with patients harboring ZNRF3 genomic loss. Heatmaps below show clinico-molecular features (ZNRF3 Loss, BCR at any time, BCR within 30 months of treatment, Metastasis, ETS Fusion status, and IDC-P/CA histology; 'present' in blue, 'absent' in white, 'not available' in gray), clinical prognostic factors (Gleason Score, pre-treatment PSA, and clinical T category), and treatment type (image-guided radiotherapy; IGRT, blue or radical prostatectomy; RP, orange); B Gene Set Enrichment Analysis of TCGA tumors harboring ZNRF3 loss. Colors represent $q$-value gradient. C Metastatic relapse-free rate in CPCG patients, stratified by ZNRF3 genomic loss and CCND1 genomic gain. $p$-value from a log-rank test $\left(p=7.48 \times 10^{-23}\right)$. Colors indicate different molecular profiles in each group. D Multivariable Cox Proportional Hazards analysis of metastatic relapse in CPCG patients, stratified by ZNRF3 genomic loss, CCP/Prolaris score (continuous), clinical prognostic factors, and PGA. $p$-values from a Wald test. Error bars represent $95 \%$ confidence intervals of the reported hazard ratios. E Volcano plot of genes upregulated in TCGA tumors harboring monoallelic ZNRF3 loss. Dashed line represents cutoff of statistical significance $(q>0.05)$. Colors show genes implicated in the DREAM (blue) or WNT (red) pathways, or both pathways (orange). F Significant RNA upregulation of genes implicated in polycomb repressive complex-1 and -2 (PRC2: EZH2, EED, SUZ12; PRC1: CBX2) in TCGA tumors $(n=493)$ harboring monoallelic losses of ZNRF3.

${ }^{\star \star \star} p<0.0001 ;{ }^{\star \star} p<0.001$. $p$-values from a Mann-Whitney $U$ test. Centre of box represents the median value. Lower and upper box hinges correspond to the first and third quartile. Whiskers extend to the largest and smallest values no further than 1.5-times the Interquartile Range. Source data are provided as a Source Data file.

harboring ZNRF3 loss, we evaluated clinical outcomes in patients with ZNRF3 genomic loss, with or without amplification of CCND1. We focused on CCND1 because its gain was significantly more prevalent in mCRPC than localized disease and was itself associated with metastatic relapse of localized disease; Fig. 2A and Table S1). While only eight patients harbored CNAs of both $Z N R F 3$ and CCND1, these patients were at significantly elevated risk of BCR and metastatic relapse $\left(p<2 \times 10^{-16}\right.$, log-rank test; Fig. 5C and S11C). To further confirm the effects of ZNRF3 and cell proliferation, we assessed the interplay between $Z N R F 3$ loss and a clinically-validated RNA-based prognostic biomarker (Prolaris/CCP), which is based on abundance of 31 genes related to cell cycle progression ${ }^{45}$. CCP score predicted risk of metastasis ( $\mathrm{HR}=1.51,95 \% \mathrm{CI}: 0.985-2.33, p=0.059$, Wald test $)$ in CPCG. However, on multivariable Cox proportional hazards analysis in CPCG, including CCP score, ZNRF3 loss, PGA, and clinical prognostic factors, only ZNRF3 loss and PGA remained prognostic of metastatic relapse at our pre-specified level of statistical significance $\left(p<0.05 ; N_{\text {METS }}=17 / 208\right.$; Fig. $\left.5 D\right)$.

GSEA also revealed strong enrichment of genes implicated in dimerization partner, RB-like, E2F and multi-vulval class B (DREAM) complex signaling and WNT activation and in Polycomb Repressive Complex-1 and -2 (PRC1/2) signaling in tumors harboring ZNRF3 loss, including EZH2, SUZ12, EED, and $C B X 2$ (Fig. 5E, F). Upregulation of PRC1/2 is strongly linked to prostate cancer progression, lineage plasticity, and prostate cancer-specific mortality ${ }^{46-50}$. Taken together, these data demonstrate a link between ZNRF3 loss and activation of pathways with well-established links to aggressive prostate cancer.

\section{Discussion}

Prostate cancer lethality is a corollary of distant metastasis. While most newly diagnosed cases are localized with no evidence of extra-glandular spread, many men relapse following apparently successful local therapy. This implies the presence of occult metastases at the time of treatment, which are not accurately predicted using standard clinical prognostic factors. We and others have used multi-omic approaches to identify candidate biomarkers that predict $\mathrm{BCR}^{7-10,51-53}$. While $\mathrm{BCR}$ is an early and easily measured endpoint, it is a weak surrogate of prostate cancer-specific mortality ${ }^{5,6}$. Assessment of more meaningful clinical endpoints (e.g. metastasis) requires mature cohorts with matched molecular data and long-term follow-up. Moreover, because large multi-omic studies interrogate many thousands of individual mutations for links to outcome, there is a significant risk of false discovery due to multiple hypothesis testing.

To overcome these limitations, we assessed differential prevalence of a set of established prostate cancer driver and recurrent mutations in localized and metastatic prostate cancer to identify candidate prognostic biomarkers of metastasis. In addition to validating established biomarkers of biochemical relapse-MYC, CCND1, and PRKDC gain, TP53 loss-as also prognostic of metastatic relapse of localized disease, we identified and validated ZNRF3 copy number loss as a prognostic biomarker of metastatic relapse of localized disease. Importantly, ZNRF3 loss is associated with higher tumor grade but provides prognostic value independently of grade and other clinical prognostic factors. Of note, neither PTEN nor RB1 losses were prognostic of metastatic relapse in CPCG, although both were prognostic of PFS in the TCGA cohort. This may be due to the reduced power of multigene studies (based on genome-wide surveys) such as this one relative to candidate gene analyses, which have previously suggested a role for these tumor suppressors in event-free survival and metastatic relapse ${ }^{54}$. Alternatively, our findings may, in part, reflect a unique biology associated with the more advanced disease enriched in the TCGA relative to the CPCG one.

WNT activation is a hallmark of mCRPC $^{21,22}$, and CNAs and hypomethylation of WNT pathway genes occur in localized prostate cancer in germline BRCA2 mutation carriers ${ }^{55,56}$, which have a significantly more aggressive clinical course than sporadic disease ${ }^{57}$. This implicates WNT signaling in the development of aggressive, potentially lethal prostate cancer, consistent with our finding-across multiple independent cohorts-of a link between ZNRF3 loss, WNT pathway activation, and adverse clinical outcomes. Similarly, the strong upregulation of cell cycle progression, E2F/DREAM, and PRC1/2 pathways in tumors harboring ZNRF3 loss further supports a link between this tumor suppressor and pathways with established links to prostate cancer aggression.

ZNRF3 genomic loss has not been identified as prognostic for overall survival in mCRPC ${ }^{22}$. Allelic gains in CTNNB1 $(26 \%$ of mCRPC specimens) were not prognostic of shorter overall survival in mCRPC. These findings are consistent with a model in which WNT pathway activation-via ZNRF3 loss, CTNNB1 allelic gains, or other mechanisms-increases the risk of metastatic spread of the primary tumor but is not required for disease progression once metastases have formed. Our finding that ZNRF3 loss or low ZNRF3 RNA abundance is associated with upregulation of cell cycle progression pathways further supports a model whereby WNT activation may promote aggressive disease in mitotically active cancers. While ZNRF3 loss is comparatively rare in localized disease, the apparent interaction of at least two mechanisms of ZNRF3 silencing (loss, hypermethylation) suggests that the detection of loss alone, may underestimate the impact of this gene as a predictor of adverse outcomes. However, longitudinal studies of patient-matched primary disease-ideally taking into account the presence of multifocal disease-and 
distant metastases will be required to establish the precise mechanisms through which these (and other) aberrations contribute to aggressive prostate cancer.

These data require careful prospective validation and testing in randomized trials but suggest several potential clinical applications for ZNRF3 loss. For example, patients with favorable intermediate risk prostate cancer whose tumors harbor ZNRF3 loss might be triaged out of active surveillance protocols and toward definitive therapy. Similarly, patients with unfavorable intermediate risk disease who would otherwise receive curativeintent $\mathrm{RP}$ or radiation might be triaged to radiation + a short course of ADT, as is now standard of care for men with high-risk disease. ZNRF3 loss has also been suggested as a potential predictive biomarker for sensitivity to porcupine inhibitors ${ }^{58}$, which are in clinical trials for WNT- and NOTCH-driven cancers (e.g. NCT01351103 $)^{59}$. Thus, while the precise clinical impact of assessing ZNRF3 loss is unclear, its presence in $\sim 10 \%$ of localized disease could make it a valuable endpoint to routinely assess. This is particularly true in the context of more frequent somatic ${ }^{60}$ or germline $^{25}$ molecular testing, where the incremental cost of assessing an additional gene would be low. Moreover, while both ZNRF3 loss and ZNRF3 RNA downregulation are significantly associated with higher grade disease, these features conferred a risk of adverse outcomes independently of grade. This suggests that assessment of ZNRF3 loss could help to identify a substantial fraction of patients who are at high risk of metastatic relapse, even amongst those with higher grade disease. Thus, in some sense the $10 \%$ overall rate of ZNRF3 loss is misleading, because it encompasses all localized disease. Those $10 \%$ of cases are strongly enriched for men at the highest risk of metastatic relapse.

Multiple lines of evidence support the hypothesis that ZNRF3 contributes to the clinical aggression observed in patients harboring monoallelic chr22q12.1 loss. Of the 9 genes in the region that were significantly associated with metastasis-free survival when downregulated at the RNA level, low ZNRF3 RNA abundance was the only one that was prognostic across four independent validation cohorts. Nevertheless, we cannot exclude the possibility that other genes in the chr22q12.1 region contribute to this aggressive phenotype. Moreover, the finding that tumors harboring >1 ZNRF3 alterations (i.e. monoallelic loss, low RNA abundance, and/or 5' hypermethylation) are significantly more aggressive than those harboring $0-1$ alterations supports a role for ZNRF3 in promoting disease aggression. These data are consistent with the hypothesis that monoallelic loss of ZNRF3 and epigenetic silencing of the remaining allele may each contribute to a reduction in ZNRF3 RNA abundance and increased tumor aggression.

The current study has some limitations; first, the microarrays used for calling CNAs in the CPCG and TCGA cohorts offer lower resolution than the sequencing-based approaches used in other cohorts. This may result in under-calling of focal CNAs leading to some driver CNAs that exhibit modestly increased prevalence in mCRPC being false positives. ZNRF3 loss was 3 -fold more prevalent in MCRPC than localized disease and was detected by microarray within a region of a minimum observed size of $\sim 1 \mathrm{Mbp}$ (median: $6 \mathrm{Mbp}$, range: 1.01-35 Mbp), though we cannot exclude the possibility that some cases may harbor focal CNAs in this region. Similarly, mutation calling based on wholegenome sequencing may have lower sensitivity than higher depth whole-exome sequencing, although the overall prevalence of SNVs in the driver genes studied was not different in wholeexome vs. whole-genome sequencing mCRPC studies (Whole Exome: $5.52 \%$; $1078 \mathrm{SNVs}$ in 454 samples $\times 43$ genes assessed per sample vs. Whole Genome: 5.39\%; 234 SNVs in 101 samples $\times 43$ genes assessed per sample; $p=0.284$, Pearson's $\mathrm{X}^{2}$ test with Yates' correction). Third, the vast majority of localized prostate cancer specimens were derived from surgically resected tissue while all mCRPC specimens were derived from biopsied metastases; the latter may have lower clonal complexity than surgical specimens. Thus, some of the mCRPC tumors may harbor subclonal mutations in driver genes which were not detected due to sampling bias. Fourth, driver SVs are under-represented across the study because several studies employed exome sequencing. Fifth, the cohorts in the study are strongly biased toward Caucasian men, and it is unclear to what extent these findings will generalize across ancestries. Sixth, the localized cohort is largely composed of intermediate risk cases. While these represent a plurality of newly diagnosed cases, it is unclear how these findings relate to other localized disease states. Finally, we cannot exclude the possibility of study-specific false-negative errors due to the use of unique analysis pipelines for each study cohort, although, the current study detected clinically relevant mutations in at least some driver genes across validated analysis pipelines ${ }^{61-63}$. For example, in localized disease, CNAs were called from either whole-genome sequencing (Gerhauser) or various microarray platforms (CPCG, TCGA, Baca, Barbieri, Taylor). ZNRF3 loss frequencies were not significantly different across these cohorts (WGS: $5 / 48$ vs. array: $108 / 1038 ; p=0.811$, Pearson's $X^{2}$ test with Yates' correction), as might be expected if detection sensitivity were inflating the CNA frequency in WGS-based studies.

Comparison of primary and metastatic tumor genomics provides an attractive strategy for prognostic biomarker discovery. We apply it to identify ZNRF3 as a predictor of metastatic relapse in localized prostate cancer. Pre-treatment evaluation of ZNRF3 tumor genomic loss and RNA abundance might improve treatment stratification for men with localized prostate cancer.

\section{Methods}

Patient cohorts, pathology, and tissue procurement. Patients in the Canadian Prostate Cancer Genome Network (CPCG) cohort (Supplementary Data 1; $n=385$ ) were consented for whole-genome sequencing and other molecular analyses and for reporting of anonymized clinical data, with approval from local Research Ethics Boards (UHN \#11-0024 and 06-0822; CHUQ 2012-913:H12-03192). All patients had National Comprehensive Cancer Network (NCCN) intermediate risk prostate cancer, were treated with radical prostatectomy (RP) or external-beam image-guided radiotherapy (IGRT) and were hormone- and chemotherapy-naïve at the time of treatment. Whole-blood or buffy coat specimens were acquired at the time of consenting. For patients undergoing RP, a freshfrozen specimen was obtained from the index lesion within the resected prostate. For patients undergoing IGRT, an ultrasound-guided biopsy to the index lesion was obtained prior to the start of radiotherapy and was flash frozen in optimal cooling temperature (OCT) medium. For all specimens, $20 \times 0 \mu \mathrm{m}$ sections were acquired, with a hematoxylin and eosin (H\&E)-stained $5 \mu \mathrm{m}$ section on the top and bottom, as well as between the 10th and 11th section, to confirm continuity of histological features. All specimens were independently audited by two urogenital pathologists for Gleason/ISUP grade ${ }^{7}$, tumor cellularity, and presence of intraductal carcinoma of the prostate (IDC-P) and cribriform architecture (CA) histology. Specimens of at least $70 \%$ tumor cellularity were used for molecular analyses. Genomic DNA was extracted using phenol:chloroform, as previously reported $^{7,10}$. Double-stranded DNA quantity was assessed using a Qubit fluorometer and quality was assessed using a Nanodrop spectrophotometer and a BioRad Bioanalyzer, as previously reported ${ }^{7}$. All clinical, pathological, and molecular data for the CPCG cohort have been reported elsewhere ${ }^{7,15,43,51,64}$.

Molecular, clinical, and pathologic data for patients in the TCGA PRAD cohort (PanCancer Atlas; $n=494$; Supplementary Data 1$)^{19}$ were obtained from cBioPortal ${ }^{65,66}$ (www.cbioportal.org) and the NIH Genomic Data Commons Data Portal (https://portal.gdc.cancer.gov). Similar data were obtained from the publicly available Baca $^{11}$, Berger ${ }^{12}$, Weischenfeldt ${ }^{13}$, Barbieri ${ }^{20}$, Gerhauser $^{14}$, Robinson ${ }^{21}$, and $\mathrm{Abida}^{22}$ cohorts (Supplementary Data 1 ). Where an individual patient sample was included in multiple reports (e.g. samples from the Baca, Berger, Barbieri, Weischenfeldt, and TCGA studies were included in a meta-analysis along with CPCG samples 7 , the Robinson/Abida SU2C mCPRC cohorts ${ }^{21,22}$ contained 125 patients that were analyzed in both studies), all cases were audited using original sample and patient identifiers across studies to ensure no duplication of patients. Data from the Quigley cohort ${ }^{18}$ was downloaded from the authors' website, as described below. Data for the Taylor/MSKCC validation cohort ${ }^{27}$ was obtained from cBioPortal.

Patients in the Mt Sinai Hospital cohort ('Mt Sinai'; $n=47$ ) were consented for molecular analysis and reporting of anonymized clinical data, with approval from 
the Research Ethics Board at Mt. Sinai Hospital and the Lunenfeld Research Institute (MSH REB \#14-0211-E and University of Toronto REB \#35275). All patients underwent RP for localized high-volume intermediate risk or high-risk prostate cancer and total RNA was extracted from fresh-frozen specimens (see below).

The final cohort for molecular discovery consisted of 1844 unique patient samples from the Abida, Baca, Barbieri, Berger, CPCG, Gerhauser, Quigley, Robinson, TCGA, and Weischenfeldt studies (Supplemenary Data 1). For clinical outcome analyses (see below), CPCG was used for discovery, with validation in the Gerhauser (EOPC), Mt. Sinai (LTRI), Taylor, and TCGA cohorts.

\section{Selection of driver aberrations. A list of 113 mutations across 72 established} driver genes or recurrently altered loci was compiled as the union of drivers reported in the Quigley and Fraser studies 7,18 (Supplementary Data 2). Mutations included copy number aberrations (CNAs), coding single nucleotide variants (SNVs; non-synonymous, stop codon gained, stop codon lost, and splice gain/loss), non-coding single nucleotide variants (ncSNVs) and non-CNA structural variants (translocations, fusions, inversions; SVs). While frameshift indels can also result in stop codon losses, we did not include these mutations in the current analysis, which focused only on established driver mutations. Mutations in the androgen receptor enhancer (624 kb upstream of $A R^{18}$; CNAs only) were pooled with those in the $A R$ gene body. For ncSNV drivers identified in $\mathrm{CPCG}^{7}$, hg19 coordinates were mapped to GRCh38 using the NCBI Remap web interface (https://www.ncbi.nlm.nih.gov/ genome/tools/remap) to compare across cohorts. In cases where a gene was subject to multiple mutation types (e.g. TP53 SNVs, CNAs, and SVs), each mutation was analyzed independently. Because not all mutation types were available for all cohorts, the denominator was different for different types:

Coding SNV: 1204 localized $+555 \mathrm{mCRPC}=1759$ specimens

Non-Coding SNV: 201 localized $+101 \mathrm{mCRPC}=302$ specimens

CNA: 1279 localized $+555 \mathrm{mCRPC}=1834$ specimens

SV: 201 localized $+101 \mathrm{mCRPC}=302$ specimens

DNA copy number aberrations. For the CPCG cohort, copy number aberrations (CNAs) were called from OncoScan FFPE v3 microarrays $(n=382)$, as previously described $^{7}$. For The Cancer Genome Atlas Prostate Adenocarcinoma (TCGAPRAD; $n=494)$ and Baca $(n=53)$ cohorts, CNAs were called from Illumina SNP 6.0 microarrays. For the Taylor/MSKCC validation cohort $(n=194)$, CNAs were called from Agilent $244 \mathrm{~K}$ array comparative genomic hybridization (aCGH) microarrays. Overall, CNA calls were available for 1279/1289 localized patients. For mCRPC specimens from the Quigley study $(n=101)$, CNAs were called from whole-genome sequencing $(n=101)$ using CopyCat-generated BED files downloaded from the authors' website (http://davidquigley.com/prostate.html) with copy number status ('gain', 'neutral', or 'loss') assigned based on $\log _{2}$ ratios according to the authors' guidelines ${ }^{18}$ : for autosomal genes, gain: $\log _{2} \mathrm{CN}$ score $\geq 3$, loss: $\log _{2}$ CN score $\leq 1.65$, neutral: $1.65<\log _{2}$ CN score $<3$; for genes on chromosomes $\mathrm{X}$ and $\mathrm{Y}$, gain: $\log _{2} \mathrm{CN}$ score $\geq 1.4$; loss: $\log _{2} \mathrm{CN}$ score $\leq 0.6$; neutral: $0.6<\log _{2} \mathrm{CN}$ score $<1.4$. For the Abida and Robinson mCRPC cohorts $(n=454)$, CNAs were called from whole-exome sequencing, as described ${ }^{21}$. In all cases except CPCG and Quigley cohorts, CNA data were extracted from publicly available datasets via the CGDS-R package ( $\mathrm{v}$ 1.3.0). In these cases, shallow deletion and deep deletion were pooled as loss while gain and amplification were pooled as gain.

Percentage of the genome affected by a copy number alteration (PGA) was calculated as the number of bases affected by a CNA divided by the total number of bases in the genome, as previously reported ${ }^{7,32,67,68}$. When comparing the PGA associated with the presence or absence of a specific CNA, an adjusted PGA was calculated by omitting the chromosome on which the specific gene is found.

Single nucleotide variants. Coding single nucleotide variants (SNVs) in driver genes were called from whole-genome or whole-exome sequencing data based on tumor-normal comparisons. Of the 1289 localized prostate cancer patients, coding SNV data were available from 1204 independent localized patient specimens (CPCG: 300, Barbieri: 109, Berger: 7, Baca: 53, TCGA: 494, Weischenfeldt: 11, and Gerhauser: 230) and 555 mCRPC patient specimens (Quigley: 101, SU2C: 454). As noted above, these numbers represent unique patients; where a given specimen was included in two or more studies, it was included only once in the current study. SNV data for all localized prostate cancer studies were downloaded from cBioPortal (via the CGDS-R package for R) or the ICGC Data Portal (dcc.icgc.org). For Quigley mCRPC specimens, VCF files were downloaded from the authors' website (http://davidquigley.com/prostate.html). For Robinson and Abida cohort mCRPC specimens, SNV calls were downloaded into R from cBioPortal using the CGDS-R package (v1.3.0). For each patient, we extracted calls for missense (non-synonymous), nonsense (stop codon gained or stop codon lost), and splicing variants (spice donor or splice acceptor) within each gene analyzed.

For non-coding SNVs in the Quigley cohort, hg19 coordinates from the CPCG study were re-mapped to GRCh38 (as described above).

The list of genes affected by SNVs in the current study is available in Supplementary Data 2. Amongst the 43 genes evaluated for SNVs, 20 were included in a previous analysis of driver SNV enrichment in localized prostate cancer vs. mCRPC ${ }^{16}$.
Structural variants. Driver structural variants (SVs) in the CPCG cohort were previously reported ${ }^{7}$. For mCRPC specimens, SV calls from Manta ${ }^{69}$ were downloaded from the authors' website (http://davidquigley.com/prostate.html). Overall, SV calls were available for 201 localized patients and 101 mCRPC patients. SVs included translocations and inversions, except where a specific SV type is specified for a given gene or locus. To assess SVs that were originally called from localized specimens in one megabase bins based on hg19 coordinates, these bins were remapped to GRCh38 using the NCBI Genome Remapping Service (https:// www.ncbi.nlm.nih.gov/genome/tools/remap). For SVs at the PTEN locus, we evaluated inter-chromosomal translocations and deletions separately from inversions within the chr10:89 Mbp bin (hg19), which regulate PTEN RNA abundance in localized prostate cancer ${ }^{7}$.

RNA abundance data. RNA abundance data were available for 208 patients with clinical outcome data in the CPCG cohort ${ }^{51}$. For all cases, total RNA was extracted from tumor tissue sections, alternating with those used for whole-genome sequencing to minimize any effects of spatial heterogeneity. Total RNA (100 ng) was assayed using Affymetrix Human Transcriptome Array 2.0 and HuGene 2.0 ST microarrays, and RNA abundance calculated as previously reported ${ }^{7,10}$. Samples were stratified as having high or low RNA abundance based on median dichotomization of $\log _{2}$ abundance values.

The LHRI cohort RNA abundance was assessed from RNA-seq $(n=47)$. Briefly, total RNA was extracted using RNeasy Mini kit (Qiagen, Maryland, USA). RNA quantity was assessed using a Qubit fluorometer (ThermoFisher,

Massachusetts, USA). $200 \mathrm{ng}$ of total RNA was used to construct a TruSeq strand specific library with the Ribo-Zero protocol (Illumina), and all samples were sequenced on a HiSeq2500 to a target depth of 50 million read pairs. Reads were mapped and mRNA abundance was quantified using the STAR aligner (v2.5.2a) against GRCh37 with Gencode Annotations (v24) lifted to GRCh $37^{70}$. Library normalization was performed using trimmed means of M-values (TMM) with the BioConductor package EdgeR (v3.12.1 $)^{71}$. Samples were stratified as having high or low RNA abundance based on median dichotomization of $\log _{2}$ abundance.

RNA abundance data for TCGA (RNA-seq; $n=493$ ) and Taylor/MSKCC (Affymetrix Human Exon 1.0 ST microarrays; $n=216$ ) were downloaded into R from cBioPortal (www.cBioPortal.org) using CGDS-R (v1.3.0). To take advantage of the increased statistical power from these larger cohorts, we stratified samples into quartiles based on $\log _{2}$ RNA abundance values.

Comparison of RNA abundance between groups was performed using the Spearman rank correlation coefficients and Mann-Whitney U tests. Gene Set Enrichment Analysis (GSEA) and Gene Ontology (GO) analyses were performed using the online Molecular Signatures Database tool from the Broad Institute (v7.0; March 3, 2020; http://software.broadinstitute.org/gsea/msigdb/annotate.jsp) an G:Profiler g:GOSt (version e99_eg46_p14_f929183d, July 20, 2020; https:// biit.cs.ut.ee/gprofiler/).

Comparison of driver aberration prevalence. For the localized and mCRPC cohorts (as well as the two cohorts combined), we calculated the proportion of samples harboring mutations in each driver gene (e.g. TP53) as well as the proportion of samples harboring each driver gene mutation type (e.g. TP53 SNVs, CNAs, and SVs). For each individual mutation class (CNA, SNV, or SV), the proportion $\left(P_{\mathrm{MUT}}\right)$ was calculated as

$$
P_{\mathrm{MUT}}=\frac{\text { number of specimens with specific mutation }}{\text { number of specimens analysed for mutation class }}
$$

We also calculated the proportion of specimens harboring more than one mutation class (i.e. CNA + SNV, CNA + SV, SNV + SV, and/or CNA + SNV + SV). $95 \%$ confidence intervals for the proportions of each mutational class (or combination of classes) were calculated as:

$$
\mathrm{CI}=p \pm 1.96 \sqrt{p(1-p) / n}
$$

where $p$ is the overall proportion for each mutation or combination of mutations for each gene and $n$ is the number of samples analyzed for that mutation class or combination of classes.

For each gene, the final proportion of cases harboring a mutation of any kind $\left(P_{\mathrm{GENE}}\right)$ was calculated as

$$
P_{\mathrm{GENE}}=\left(P_{\mathrm{CNA}}+m P_{\mathrm{SNV}}+P_{\mathrm{SV}}\right)-\left(P_{\mathrm{CNA \& SNV}}+P_{\mathrm{CNA \& SV}}+P_{\mathrm{SNV} \& \mathrm{SV}}+P_{\mathrm{CNA \& SNV} \& \mathrm{SV}}\right)
$$

where $P$ is the proportion of cases harboring each stated mutation class (i.e. CNA, SNV, SV; as applicable to the specific gene). To account for unequal sample sizes for each mutation class (or combination of classes), we calculated propagation of error for each mutation class (or combination of classes, as applicable) as

$$
\mathrm{PE}_{\text {class }}=\sqrt{\mathrm{CI}_{\mathrm{Upper}^{2}}^{2}+\mathrm{CI}_{\text {Lower }}^{2}}
$$

Variance of propagation of error was calculated as

$$
\partial_{\mathrm{GENE}}^{2}=\sqrt{\left(\mathrm{PE}_{\mathrm{CNA}}+\mathrm{PE}_{\mathrm{SNV}}+\mathrm{PE}_{\mathrm{SV}}\right)-\left(\mathrm{PE}_{\mathrm{CNA \& SNV}}+\mathrm{PE}_{\mathrm{CNA} \& \mathrm{SV}}+\mathrm{PE}_{\mathrm{SNV} \& S V}+\mathrm{PE}_{\mathrm{CNA \& SNV} \& S V}\right)}
$$


Final 95\% confidence intervals for each gene were calculated as above

$$
\mathrm{CI}=p \pm 1.96 \sqrt{p(1-p) / n}
$$

where $p$ is the overall gene proportion and $n$ is the number of samples analyzed for that gene (Supplementary Data 2).

The per patient overlap in available mutation data is shown in Fig. S1. Differences in proportion of localized and mCRPC patients (' $\Delta$ proportion') were calculated by subtracting the proportion of localized specimens harboring the specific aberration from the proportion of $\mathrm{MCRPC}$ specimens harboring the same aberration. Positive $\Delta$ proportion values indicate higher prevalence in mCRPC; negative $\Delta$ proportion values indicate enrichment in localized disease. To determine if specific mutations were statistically differentially prevalent between localized disease and mCRPC, we used Fisher's Exact tests. For SNVs (coding and non-coding), we corrected for global mutational burden as follows: first, we used a binomial distribution to calculate the probability of observing no mutations in a given driver gene in a given sample, corrected for the size of the gene coding region $(\mathrm{Mbp})$ and the mutational burden (SNVs per Mbp) in that sample $\left(P_{\text {NOMUT }}\right)$; the probability of observing at least one mutation in a given gene was then calculated as: $P_{\mathrm{MUT}}=1-P_{\mathrm{NOMUT}}$. We then performed a simulation of 100,000 samples of the binomial distribution, using $P_{\mathrm{MUT}}$ to weight the probability of mutation in each sample. A two-sided $p$-value was calculated as proportion of these simulations showing as great or greater simulated $\Delta$ proportion than observed $\Delta$ proportion.

We corrected for CNA burden in a similar manner, using gene size and per sample CNA burden (i.e. proportion of the genome altered by a $\mathrm{CNA} \times 3 \times 10^{9}$ ) to identify $P_{\text {MUT }}$ (as above), which was then used to weight the sampling probability. We then calculated a two-sided $p$-value for each driver CNA as half of the proportion of permutations showing as great or greater simulated $\Delta$ proportion than the observed $\Delta$ proportion (to account for the fact that a CNA can be either a gain or a loss).

Confidence intervals for $\Delta$ proportions were calculated using Yates' $\mathrm{X}^{2}$ with continuity correction,

$$
\mathrm{CI}=\hat{p}_{1}-\hat{p}_{2} \pm 1.96 \sqrt{\frac{\hat{p}_{1}\left(1-\hat{p}_{1}\right)}{m}+\frac{\hat{p}_{2}\left(1-\hat{p}_{2}\right)}{n}} \pm \frac{1}{2} *\left(\frac{1}{m}+\frac{1}{n}\right)
$$

where $p_{1}$ is the proportion of $\mathrm{mCRPC}$ harboring the mutation, $p_{2}$ is the proportion in localized prostate cancer harboring the mutation, $m$ is the number of mCRPC specimens tested, $n$ is the number of localized prostate cancer specimens tested.

Adjusted $\Delta$ proportion was calculated as the difference between observed $\Delta$ proportion and expected $\Delta$ proportion; adjusted $\Delta$ proportion values $>0$ indicate a higher-than-expected proportion of mCRPC cases harboring the specific mutation while adjusted $\Delta$ proportion $<0$ indicates a higher-than-expected proportion in localized disease.

Clinical outcome. For patients undergoing RP, biochemical relapse (BCR) was defined according to American Urological Association (AUA) guidelines: two consecutive PSA values of $>0.2 \mathrm{ng} / \mathrm{mL}$ over at least 6 months following treatment or initiation of salvage therapy. Patients who had initial PSA failure after RP and then underwent successful salvage RT (i.e. PSA $<0.2 \mathrm{ng} / \mathrm{mL}$ in two consecutive tests within 6 months) were not classified as having BCR unless they subsequently met AUA (not Phoenix) conditions for BCR; in these cases, BCR was backdated to the time of initial post-RP PSA rise. For patients who underwent IGRT with curative intent, BCR was defined according to the Phoenix criteria ${ }^{72}$ : a PSA value of $2 \mathrm{ng} / \mathrm{mL}$ above PSA nadir or initiation of salvage hormone therapy. For the TCGA cohort, progression-free, disease-specific, and overall survival were used as reported by the consortium ${ }^{73}$.

Biochemical relapse-free rate (bRFR), metastatic relapse-free rate (mRFR) and overall survival were calculated using the Kaplan-Meier method. Associations between mutations and outcome were assessed using log-rank or univariate Cox proportional hazards models, as appropriate. Adjustments for clinical factors [Tcategory (categorical; T1, T2a/b, or T2c), pre-treatment PSA, and diagnostic (i.e. biopsy) ISUP grade (categorical; Grade 1 vs. Grade 2 vs. $\geq$ Grade 3)] using multivariable Cox proportional hazards modeling. In all cases, the proportional hazards assumptions were verified graphically using Schoenfeld residuals. Log-rank tests were used when the proportional hazards assumptions were violated.

Prognostic signature scores. Fraser signature scores were calculated based on a modified version of the six-feature signature reported in Fraser et al. ${ }^{7}$. Briefly, MYC gain, ATM SNVs, TCERGL1 hypomethylation, ACTL6B hypermethylation, chr7:61 $\mathrm{Mbp}$ inter-chromosomal translocations, and clinical $\mathrm{T}$ category were scored for each patient; CNAs, SNVs, and SVs were scored as absent (0) or present (1); probe-based methylation $\beta$-values were median dichotomized and patients scored as being either above or below the median; clinical T category was scored as cT1 or cT2a/b $(0)$ or cT2c (1). For multivariable analyses, a signature score was derived based on the sum of the six features. For visualization, scores were median dichotomized and patients assigned to either "Signature High" or "Signature Low" bins.

Cell Cycle Progression/Prolaris scores were approximated as previously reported ${ }^{45,74}$. Briefly, the mean abundance of the $31 \mathrm{CCP}$ genes was normalized to the mean abundance of 15 housekeeping genes to yield a CCP score, as previously reported ${ }^{45}$. Outcomes analyses were performed using CCP as a continuous variable.
Differential DNA methylation analyses. Illumina HumanMethylation 450k microarray data for CPC-GENE were pre-processed as previously described ${ }^{7}$. TCGA PRAD 450k data were obtained from the TCGA data portal (https:// portal.gdc.cancer.gov/projects/TCGA-PRAD). Beta values (i.e. ratio of methylated to unmethylated signal) were transformed to M-values (i.e. $\log _{2}$ ratio of methylated probes to unmethylated probes) using the beta $2 \mathrm{~m}$ function within the lumi $\mathrm{R}$ package (v. 2.24.0) ${ }^{75}$. For each cohort, patients were classified as having high or low ZNRF3 RNA abundance, as described above. Differential methylation was assessed using the limma R package (v3.13) ${ }^{76}$, including array probes mapping within genes $(n=335,926)$. Differentially methylated probes were visualized using Manhattan plots, with statistical significance inferred using a Bonferroni cutoff of $p<1.49 \times 10^{-7}$. To test the association between methylation and clinical outcomes, $M$-values for each patient were dichotomized as high or low based on Youden's J statistic, using the cutpointr R package (v1.1.1 ${ }^{77}$. Patients were then binned based on this cutoff and time-to-event (metastasis or disease progression) was determined using Cox proportional hazards models, as above.

Statistical testing and data visualization. All statistical analyses were performed using R statistical software (v3.5.2) with the following packages: BoutrosLab.plotting.general (v5.9.2) ${ }^{78}$, VennDiagram (v1.6.20) $)^{79}$, CGDS-R (v1.3.0), survival (v2.43-3), ggplot2 (v3.1.0), easyGgplot2 (v1.0), survminer (v0.4.3), forestplot (v.1.7.2), factorial2x2 (v0.2.0), cowplot (v1.1.0) and tidyverse (v1.2.1). All tests of statistical significance were two-sided. $p$-values were corrected for multiple comparisons using the Benjamini-Hochberg False Discovery Rate (FDR) method or the Bonferroni method, as noted. For Analysis of Variance (ANOVA), between group significance was evaluated using Tukey post-hoc tests. All source code is available from Zenodo ${ }^{80}$.

Reporting summary. Further information on research design is available in the Nature Research Reporting Summary linked to this article.

\section{Data availability}

The genomic variant calls, clinical, and pathology data for the CPC-GENE study are available through the ICGC Data Portal under accession PRAD-CA. Raw whole-genome sequencing data for the CPC-GENE study are available through the European GenomePhenome Archive used accession ID EGAD00001003761. Raw DNA methylation data are available from the NCBI Gene Expression Omnibus under accession number GSE84043. Summary DNA methylation data are available from Figshare (https://doi.org/10.6084/ m9.figshare.16574486.v1). Raw and processed mRNA array data for the CPC-GENE study are available from the NCBI Expression Omnibus under accession number GSE107299. Genomic variant calls, mRNA abundance, clinical, and pathology data are available from cBioPortal for the Abida (accession prad_su2c_2019), Barbieri (accession prad_broad), Baca (accession prad_broad_2013), Robinson (accession prad_su2c_2015), Taylor (accession prad_mskcc), Gerhauser and Weischenfeldt studies (accession prostate_dkfz_2018). Raw sequencing data, genomic variant calls, mRNA abundance, and DNA methylation data for the TCGA study are available from cBioPortal (accession prad_tcga_pan_can_atlas_2018) and the GDC Data Portal (accession TCGA-PRAD). Processed genomic variant, mRNA abundance, and clinical/pathology data for the Quigley study are available from the authors website (https://quigleylab.ucsf.edu/data). Processed mRNA abundance, and clinical/ pathology data for the LTRI cohort are available from Zenodo (https://doi.org/10.5281/ zenodo.5389194). Patients in the LTRI cohort were not specifically consented for deposition of their raw sequencing data into public repositories. Therefore, raw RNAseq data for the LTRI cohort are available upon request from Dr. Michael Fraser (michael.fraser@cpcgene. com) and are subject to the requestor entering into a Data Sharing Agreement with the Lunenfeld-Tanenbaum Research Institute. We will attempt to make data available within one month of any request. Source data are provided as a Source Data file. Source data are provided with this paper.

\section{Code availability}

Scripts used for data analysis and visualization are available from Zenodo (https:// doi.org/10.5281/zenodo.5389194) and GitHub (https://github.com/mfraser3/ ZNRF3_2021).

Received: 21 May 2021; Accepted: 8 October 2021; Published online: 29 October 2021

\section{References}

1. Zhou, C. K. et al. Prostate cancer incidence in 43 populations worldwide: an analysis of time trends overall and by age group. Int. J. Cancer 138, 1388-1400 (2016).

2. Beesley, L. J. et al. Individual and population comparisons of surgery and radiotherapy outcomes in prostate cancer using Bayesian multistate models. JAMA Netw. Open 2, e187765 (2019).

3. Albertsen, P. C., Hanley, J. A., Penson, D. F., Barrows, G. \& Fine, J. 13-year outcomes following treatment for clinically localized prostate cancer in a population based Cohort. J. Urol. 177, 932-936 (2007). 
4. D'Amico, A. V. et al. Biochemical outcome after radical prostatectomy, external beam radiation therapy, or interstitial radiation therapy for clinically localized prostate cancer. JAMA 280, 969-974 (1998).

5. Jackson, W. C. et al. Intermediate endpoints after postprostatectomy radiotherapy: 5-year distant metastasis to predict overall survival. Eur. Urol. 0 , 413-419 (2018).

6. Buyyounouski, M. K., Pickles, T., Kestin, L. L., Allison, R. \& Williams, S. G. Validating the interval to biochemical failure for the identification of potentially lethal prostate cancer. J. Clin. Oncol. 30, 1857-1863 (2012).

7. Fraser, M. et al. Genomic hallmarks of localized, non-indolent prostate cancer. Nature 541, 359-364 (2017).

8. Wedge, D. C. et al. Sequencing of prostate cancers identifies new cancer genes, routes of progression and drug targets. Nat. Genet. 50, 682-692 (2018).

9. Cooper, C. S. et al. Analysis of the genetic phylogeny of multifocal prostate cancer identifies multiple independent clonal expansions in neoplastic and morphologically normal prostate tissue. Nat. Genet. 47, 367-372 (2015).

10. Boutros, P. C. et al. Spatial genomic heterogeneity within localized, multifocal prostate cancer. Nat. Genet. 47, 736-745 (2015).

11. Baca, S. C. et al. Punctuated evolution of prostate cancer genomes. Cell 153, 666-677 (2013).

12. Berger, M. F. et al. The genomic complexity of primary human prostate cancer. Nature 470, 214-220 (2011).

13. Weischenfeldt, J. et al. Integrative genomic analyses reveal an androgen-driven somatic alteration landscape in early-onset prostate cancer. Cancer Cell 23, 159-170 (2013).

14. Gerhauser, C. et al. Molecular evolution of early-onset prostate cancer identifies molecular risk markers and clinical trajectories. Cancer Cell 34, 996-1011.e8 (2018).

15. Espiritu, S. M. G. et al. The evolutionary landscape of localized prostate cancers drives clinical aggression. Cell 173, 1003-1013.e15 (2018).

16. Armenia, J. et al. The long tail of oncogenic drivers in prostate cancer. Nat. Genet. 50, 645-651 (2018)

17. van Dessel, L. F. et al. The genomic landscape of metastatic castrationresistant prostate cancers reveals multiple distinct genotypes with potential clinical impact. Nat. Commun. 10, 1-13 (2019)

18. Quigley, D. A. et al. Genomic Hallmarks and structural variation in metastatic prostate. Cancer Cell 174, 758-769.e9 (2018).

19. Cancer Genome Atlas Research, N. et al. The molecular taxonomy of primary prostate cancer. Cell 163, 1011-1025 (2015).

20. Barbieri, C. E. et al. Exome sequencing identifies recurrent SPOP, FOXA1 and MED12 mutations in prostate cancer. Nat. Genet. 44, 685-689 (2012).

21. Robinson, D. et al. Integrative clinical genomics of advanced prostate cancer. Cell 161, 1215-1228 (2015).

22. Abida, W. et al. Genomic correlates of clinical outcome in advanced prostate cancer. Proc. Natl Acad. Sci. USA 116, 11428-11436 (2019).

23. Grasso, C. S. et al. The mutational landscape of lethal castration-resistant prostate cancer. Nature 487, 239-243 (2012).

24. Mateo, J. et al. DNA-repair defects and olaparib in metastatic prostate cancer. N. Engl. J. Med. 373, 1697-1708 (2015).

25. Pritchard, C. C. et al. Inherited DNA-repair gene mutations in men with metastatic prostate cancer. N. Engl. J. Med. 375, 443-453 (2016).

26. Boysen, G. et al. SPOP mutated/CHD1 deleted lethal prostate cancer and abiraterone sensitivity. Clin. Cancer Res. https://doi.org/10.1158/10780432.CCR-18-0937 (2018).

27. Taylor, B. S. et al. Integrative genomic profiling of human prostate cancer. Cancer Cell 18, 11-22 (2010).

28. Ishkanian, A. S. et al. High-resolution array CGH identifies novel regions of genomic alteration in intermediate-risk prostate cancer. Prostate 69, 1091-1100 (2009).

29. Goodwin, J. F. et al. DNA-PKcs-mediated transcriptional regulation drives prostate cancer progression and metastasis. Cancer Cell 28, 97-113 (2015).

30. Chung, J. H. et al. Prospective comprehensive genomic profiling of primary and metastatic prostate tumors. JCO Precis. Oncol 3, 1-23 (2019).

31. Ding, Z. et al. SMAD4-dependent barrier constrains prostate cancer growth and metastatic progression. Nature 470, 269-273 (2011).

32. Lalonde, E. et al. Tumour genomic and microenvironmental heterogeneity for integrated prediction of 5-year biochemical recurrence of prostate cancer: a retrospective cohort study. Lancet Oncol. 15, 1521-1532 (2014).

33. Gerhauser, C. et al. Molecular evolution of early-onset prostate cancer identifies molecular risk markers and clinical trajectories cancer cell article molecular evolution of early-onset prostate cancer identifies molecular risk markers and clinical trajectories. Cancer Cell 34, 996-1011.e8 (2018).

34. Fraser, M. Supplementary Data. Zenodo Dataset. https://doi.org/10.5281/ zenodo.5389194 (2021)

35. Chua, M. L. K. et al. A prostate cancer "Nimbosus": genomic instability and SChLAP1 dysregulation underpin aggression of intraductal and cribriform subpathologies. Eur. Urol. 72, 665-674 (2017).
36. Van Der Kwast, T. et al. Biopsy diagnosis of intraductal carcinoma is prognostic in intermediate and high risk prostate cancer patients treated by radiotherapy. Eur. J. Cancer 48, 1318-1325 (2012)

37. Chandran, U. R. et al. Gene expression profiles of prostate cancer reveal involvement of multiple molecular pathways in the metastatic process. $B M C$ Cancer 7, 64 (2007).

38. Fraser, M. CPCG_Methylation_BValues.rds. Figshare Dataset (2021) https:// doi.org/10.6084/m9.figshare.16574486.v1 (2021).

39. Siadat, F. et al. Not all gleason pattern 4 prostate cancers are created equal: a study of latent prostatic carcinomas in a cystoprostatectomy and autopsy series. Prostate 75, 1277-1284 (2015).

40. Trudel, D. et al. Prognostic impact of intraductal carcinoma and large cribriform carcinoma architecture after prostatectomy in a contemporary cohort. Eur. J. Cancer 50, 1610-1616 (2014).

41. Kweldam, C. F. et al. Cribriform growth is highly predictive for postoperative metastasis and disease-specific death in Gleason score 7 prostate cancer. Mod. Pathol. 28, 457-464 (2015).

42. Kweldam, C. F. et al. Presence of invasive cribriform or intraductal growth at biopsy outperforms percentage grade 4 in predicting outcome of Gleason score $3+4=7$ prostate cancer. Mod. Pathol. 30, 1126-1132 (2017).

43. Bhandari, V. et al. Molecular landmarks of tumor hypoxia across cancer types. Nat. Genet. 51, 308-318 (2019).

44. Milosevic, M. et al. Tumor hypoxia predicts biochemical failure following radiotherapy for clinically localized prostate cancer. Clin. Cancer Res. https:// doi.org/10.1158/1078-0432.CCR-11-2711 (2012).

45. Cuzick, J. et al. Prognostic value of an RNA expression signature derived from cell cycle proliferation genes in patients with prostate cancer: a retrospective study. Lancet Oncol. 12, 245-255 (2011).

46. Chase, A. \& Cross, N. C. P. Aberrations of EZH2 in cancer. Clin. Cancer Res. 17, 2613-2618 (2011).

47. Nuytten, M. et al. The transcriptional repressor NIPP1 is an essential player in EZH2-mediated gene silencing. Oncogene 27, 1449-1460 (2008).

48. Dardenne, E. et al. N-Myc induces an EZH2-mediated transcriptional program driving neuroendocrine prostate cancer. Cancer Cell 30, 563-577 (2016).

49. Yu, J. et al. An integrated network of androgen receptor, polycomb, and TMPRSS2-ERG gene fusions in prostate cancer progression. Cancer Cell 17, 443-454 (2010)

50. Clermont, P. L. et al. Polycomb-mediated silencing in neuroendocrine prostate cancer. Clin. Epigenetics 7, 40 (2015).

51. Sinha, A. et al. The proteogenomic landscape of curable prostate cancer. Cancer Cell 35, 414-427.e6 (2019).

52. Kron, K. J. et al. DNA methylation of HOXD3 as a marker of prostate cancer progression. Lab Invest. 90, 1060-1067 (2010).

53. Olkhov-Mitsel, E. et al. Distinct DNA methylation alterations are associated with cribriform architecture and intraductal carcinoma in Gleason pattern 4 prostate tumors. Oncol. Lett. 14, 390-396 (2017).

54. Hamid, A. A. et al. Compound genomic alterations of TP53, PTEN, and RB1 Tumor suppressors in localized and metastatic prostate cancer. Eur. Urol. $\mathbf{7 6}$, 89-97 (2019)

55. Taylor, R. A. et al. The influence of BRCA2 mutation on localized prostate cancer. Nat. Rev. Urol. https://doi.org/10.1038/s41585-019-0164-8 (2019).

56. Taylor, R. A. et al. Germline BRCA2 mutations drive prostate cancers with distinct evolutionary trajectories. Nat. Commun. 8, 13671 (2017).

57. Risbridger, G. P. et al. Patient-derived xenografts reveal that intraductal carcinoma of the prostate is a prominent pathology in brca2 mutation carriers with prostate cancer and correlates with poor prognosis. Eur. Urol. 67, 496-503 (2015)

58. Liu, J. et al. Targeting Wnt-driven cancer through the inhibition of Porcupine by LGK974. Proc. Natl Acad. Sci. USA 110, 20224-20229 (2013).

59. Rodon, J. et al. Phase 1 study of single-agent WNT974, a first-in-class Porcupine inhibitor, in patients with advanced solid tumours. $\mathrm{Br}$. J. Cancer 1251, 28-37 (2021).

60. Feng, F. Y. et al. Validation of a 22-gene genomic classifier in patients with recurrent prostate cancer. JAMA Oncol. https://doi.org/10.1001/ jamaoncol.2020.7671 (2021).

61. Bailey, M. H. et al. Retrospective evaluation of whole exome and genome mutation calls in 746 cancer samples. Nat. Commun. 11, 17 (2020).

62. Liu, L. Y. et al. Quantifying the influence of mutation detection on tumour subclonal reconstruction. Nat. Commun. 11, 1-15 (2020).

63. Campbell, P. J. et al. Pan-cancer analysis of whole genomes. Nature 578, 82-93 (2020).

64. Houlahan, K. E. et al. Genome-wide germline correlates of the epigenetic landscape of prostate cancer. Nat. Med. 25, 1615-1626 (2019).

65. Cerami, E. et al. The cBio Cancer Genomics Portal: an open platform for exploring multidimensional cancer genomics data. Cancer Discov. https:// doi.org/10.1158/2159-8290.CD-12-0095 (2021). 
66. Gao, J. et al. Integrative analysis of complex cancer genomics and clinical profiles using the cBioPortal. Sci. Signal. https://doi.org/10.1126/ scisignal.2004088 (2021).

67. Lalonde, E. et al. Translating a prognostic DNA genomic classifier into the clinic: retrospective validation in 563 localized prostate tumors. Eur. Urol. 72, 22-31 (2017).

68. Hieronymus, H. et al. Tumor copy number alteration burden is a pan-cancer prognostic factor associated with recurrence and death. Elife 7, e3729 (2018).

69. Chen, X. et al. Manta: rapid detection of structural variants and indels for germline and cancer sequencing applications. Bioinformatics 32, 1220-1222 (2016).

70. Harrow, J. et al. GENCODE: The reference human genome annotation for the ENCODE project. Genome Res. 22, 1760-1774 (2012).

71. Robinson, M. D., McCarthy, D. J. \& Smyth, G. K. edgeR: a Bioconductor package for differential expression analysis of digital gene expression data. Bioinformatics 26, 139-140 (2010).

72. Roach, M. 3rd et al. Defining biochemical failure following radiotherapy with or without hormonal therapy in men with clinically localized prostate cancer: recommendations of the RTOG-ASTRO Phoenix Consensus Conference. Int J. Radiat. Oncol. Biol. Phys. 65, 965-974 (2006).

73. Liu, J. et al. An integrated TCGA pan-cancer clinical data resource to drive high-quality survival outcome analytics. Cell 173, 400-416.e11 (2018).

74. Canter, D. J. et al. Analysis of the prognostic utility of the cell cycle progression (CCP) score generated from needle biopsy in men treated with definitive therapy. Prostate Cancer Prostatic Dis. https://doi.org/10.1038/ s41391-019-0159-9 (2019).

75. Du, P., Kibbe, W. A. \& Lin, S. M. lumi: a pipeline for processing Illumina microarray. Bioinformatics 24, 1547-1548 (2008).

76. Ritchie, M. E. et al. limma powers differential expression analyses for RNAsequencing and microarray studies. Nucleic Acids Res. 43, e47 (2015)

77. Thiele, C. \& Hirschfeld, G. cutpointr: improved estimation and validation of optimal cutpoints in R. SJ. Stat. Softw 98, 1-27 (2021).

78. P'ng, C. et al. BPG: Seamless, automated and interactive visualization of scientific data. BMC Bioinform. 20, 42 (2019).

79. Lam, F. et al. VennDiagramWeb: a web application for the generation of highly customizable Venn and Euler diagrams. BMC Bioinform. 17, 401 (2016).

80. Fraser, M. Code Repository. https://doi.org/10.5281/zenodo.5389194 (2021).

\section{Acknowledgements}

Thanks to Drs. Kathleen Houlahan, Viniyak Bhandari, and John Watson for assistance with experimental design and statistical analysis and to all members of the Boutros lab for suggestions and support. This study was conducted with the support of Movember funds through Prostate Cancer Canada, and with the additional support of the Ontario Institute for Cancer Research, funded by the Government of Ontario. This work was supported by funds from the Department of Surgical Oncology and the Genetics and Epigenetics program, Princess Margaret Cancer Centre and the University of Toronto, Department of Surgery (Division of Urology) to Michael Fraser. Hansen H. He is supported by a Project Grant from the CIHR. This work was supported by Prostate Cancer Canada and is proudly funded by the Movember Foundation Team Grant T2013 and \#RS2014-01 and a Prostate Cancer Canada Movember Discovery Grant (D2014-26) to Alexandre R. Zlotta. Paul C. Boutros was supported by a Terry Fox Research Institute
New Investigator Award, a Prostate Cancer Canada Rising Star Fellowship, a CIHR New Investigator Award, a CIHR Project Grant, the Government of Canada through Genome Canada and the Ontario Genomics Institute (OGI-125), Canadian Cancer Society (grant \#705649) and by the University of California. The authors gratefully thank the Princess Margaret Cancer Centre Foundation and Radiation Medicine Programme Academic Enrichment Fund for support (to Robert G. Bristow). This work was supported by a Terry Fox Research Institute Programme Project Grant. Robert G. Bristow is a recipient of a Canadian Cancer Society Research Scientist Award. Laboratory work for R.G.B. is supported by the CRUK Manchester Institute through Cancer Research UK. This work was supported by the NIH/NCI through awards P30CA016042, U01CA214194, and U24CA248265.

\section{Author contributions}

Initiated the study: M.F., R.G.B., P.C.B. Pathology audits: T.v.d.K. Patient accrual: A.F. A.R.Z., R.G.B. Data analysis: M.F., J.L., J.W. Data visualization: M.F., P.C.B. Supervised study: M.F., H.H.H., J.W., T.v.d.K., A.R.Z., R.G.B., P.C.B. Wrote the first draft of the manuscript: M.F. Approved the final manuscript: all authors.

\section{Competing interests}

The authors declare no competing interests.

\section{Additional information}

Supplementary information The online version contains supplementary material available at https://doi.org/10.1038/s41467-021-26489-0.

Correspondence and requests for materials should be addressed to Michael Fraser or Paul C. Boutros.

Peer review information Nature Communications thanks Todd Morgan and the other, anonymous reviewer(s) for their contribution to the peer review of this work. Peer reviewer reports are available.

Reprints and permission information is available at http://www.nature.com/reprints

Publisher's note Springer Nature remains neutral with regard to jurisdictional claims in published maps and institutional affiliations.

(1) Open Access This article is licensed under a Creative Common BY Attribution 4.0 International License, which permits use, sharing, adaptation, distribution and reproduction in any medium or format, as long as you give appropriate credit to the original author(s) and the source, provide a link to the Creative Commons license, and indicate if changes were made. The images or other third party material in this article are included in the article's Creative Commons license, unles indicated otherwise in a credit line to the material. If material is not included in the article's Creative Commons license and your intended use is not permitted by statutory regulation or exceeds the permitted use, you will need to obtain permission directly from the copyright holder. To view a copy of this license, visit http://creativecommons.org/ licenses/by/4.0\%

(C) The Author(s) 2021 